\title{
DISCRETE TRANSPARENT BOUNDARY CONDITIONS FOR THE SCHRÖDINGER EQUATION ON CIRCULAR DOMAINS*
}

\author{
ANTON ARNOLD ${ }^{\dagger}$, MATTHIAS EHRHARDT ${ }^{\ddagger}$, MAIKE SCHULTE $^{\S}$, AND \\ IVAN SOFRONOV
}

\begin{abstract}
We propose transparent boundary conditions (TBCs) for the time-dependent Schrödinger equation on a circular computational domain. First we derive the two-dimensional discrete TBCs in conjunction with a conservative Crank-Nicolson finite difference scheme. The presented discrete initial boundary-value problem is unconditionally stable and completely reflectionfree at the boundary. Then, since the discrete TBCs for the Schrödinger equation with a spatially dependent potential include a convolution with respect to time with a weakly decaying kernel, we construct approximate discrete TBCs with a kernel having the form of a finite sum of exponentials, which can be efficiently evaluated by recursion. In numerical tests we finally illustrate the accuracy, stability, and efficiency of the proposed method.

As a by-product we also present a new formulation of discrete TBCs for the 1D Schrödinger equation, with convolution coefficients that have better decay properties than those from the literature.
\end{abstract}

Key words. Two-dimensional Schrödinger equation, transparent boundary conditions, discrete convolution, sum of exponentials, Padé approximations, finite difference schemes.

AMS subject classifications. 65M12, 35Q40, 45K05.

\section{Introduction}

The Schrödinger equation. Consider in the circular geometry with polar coordinates $(r, \theta)$ the following Cauchy problem for the scaled transient Schrödinger equation:

$$
\begin{gathered}
i \psi_{t}=-\frac{1}{2}\left[\frac{1}{r}\left(r \psi_{r}\right)_{r}+\frac{1}{r^{2}} \psi_{\theta \theta}\right]+V(r, \theta, t) \psi, \quad r \geq 0,0<\theta \leq 2 \pi, t>0, \\
\psi(r, \theta, 0)=\psi^{I}(r, \theta), \quad r \geq 0,0<\theta \leq 2 \pi .
\end{gathered}
$$

We assume that the given $\theta$-periodical potential $V$ is constant outside of the computational domain $[0, R] \times[0,2 \pi]$ :

$$
V(r, \theta, t)=V_{R} \equiv \text { const } \quad \text { for } r \geq R,
$$

and that the sufficiently smooth $\theta$-periodical initial data has compact support:

$$
\operatorname{supp} \psi^{I} \subset[0, R) \times[0,2 \pi] .
$$

Discussions of strategies to soften these restrictions could be found in $[22,23,35$, 46]. The strategy from [23] to overcome the assumption on the compact support of

*Received: October 17, 2008; accepted (in revised version): November 18, 2011. Communicated by Shi Jin.

$\dagger$ Institut für Analysis und Scientific Computing, Technische Universität Wien, Wiedner Hauptstr. 8, 1040 Wien, Austria (anton.arnold@tuwien.ac.at).

${ }^{\ddagger}$ Lehrstuhl für Angewandte Mathematik und Numerische Analysis, Fachbereich C - Mathematik und Naturwissenschaften, Bergische Universität Wuppertal, Gaußstr. 20, 42119 Wuppertal, Germany (ehrhardt@math.uni-wuppertal.de).

$\S$ Institut für Numerische und Angewandte Mathematik, Universität Münster, Einsteinstr. 62, 48149 Münster, Germany (Maike.Schulte@math.uni-muenster.de).

『Keldysh Institute of Applied Mathematics, Russian Academy of Sciences, Miusskaya sq. 4, Moscow, Russia (sofronov@spp.keldysh.ru). 


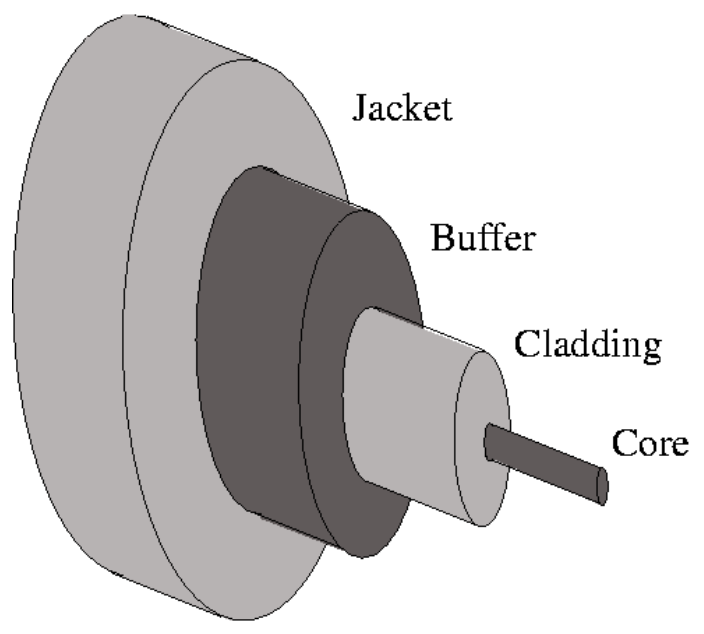

FIG. 1.1. A typical single-mode optical fiber, showing the different component layers. Wave propagation mostly takes place in the relatively thin core region.

the initial data is directly applicable to the $2 \mathrm{D}$ case considered here. However, the computational effort will increase tremendously.

In addition to quantum mechanics, Equation (1.1a) has many important applications including electromagnetic wave propagation [36], modelling of quantum devices [7], integrated optics (Fresnel equation) [45, 57], plasma physics, seismic migration [18], and (underwater) acoustics due to the paraxial approximation of the wave equation in the frequency domain $[8,56]$, etc.

One quite important application of the Schrödinger equation, especially in a circular geometry, arises in the context of wave propagation in optical fibers [32, 58]. A sketch of the structure of an optical fiber with its different layers is shown in Figure 1.1.

In modern communication networks optical fibers play a fundamental role and there it is often necessary to connect the fibers (e.g. after a breakage or to extend a cable run) with low loss. Optical fibers are connected by a fusion process called thermal splicing and one has to control this procedure and simulate how small disturbances in the geometry of the (usually straight) fiber core effect the transported light in the fiber. Doing so, one can predict the caused loss at these joining positions of the fibers.

With the proposed transparent boundary conditions in this paper one can reduce the computational domain significantly (e.g. to the core region) and obtain a fast, accurate, and reliable simulation using the beam propagation method [30, 58]. Here, the time variable $t$ corresponds to the axial variable, i.e. the propagation direction. For an adequate treatment of the density jump in the TBC between the different layers we refer to [8].

Analytic transparent boundary conditions (TBCs). Let us exemplify first analytic TBCs that can be derived for the Schrödinger equation on a circular domain. The idea is to eliminate the problem on the exterior domain $r>R$, and to replace it by a Dirichlet-to-Neumann (DtN) map. First we briefly review the construction of the analytic TBCs for the Schrödinger equation on a circular domain from [5] and extend them to the case of a nonzero potential $V_{R}$ at infinity (cf. also $[26,31]$ ). For a 
review paper about TBCs for linear and nonlinear Schrödinger equations including a (theoretical) comparison to the widely used Perfectly Matched Layer (PML) technique we refer the interested reader to [6] and to [59]. For a detailed numerical comparison of PML and discrete TBCs for the Schrödinger equation we refer to [41].

We consider sufficiently smooth bounded periodical solutions to (1.1a) on the exterior domain $r>R$ and denote by $\hat{\psi}=\hat{\psi}(r, \theta, s)$ the Laplace transform of $\psi$ with respect to time. The transformation of (1.1a) reads:

$$
\frac{1}{r}\left(r \hat{\psi}_{r}\right)_{r}+\frac{1}{r^{2}} \hat{\psi}_{\theta \theta}+2 i\left(s+i V_{R}\right) \hat{\psi}=0, \quad r \geq R, \quad 0<\theta \leq 2 \pi,
$$

where we used the assumption (1.2). We use a Fourier series with respect to the angle $\theta$ :

$$
\hat{\psi}(r, \theta, s)=\sum_{m \in \mathbb{Z}} \hat{\psi}^{(m)}(r, s) e^{i m \theta}, \quad r \geq R .
$$

Then, for each mode $m \in \mathbb{Z}$, the Fourier coefficient $\hat{\psi}^{(m)}(r, s)$ satisfies the ordinary differential equation

$$
\frac{1}{r}\left(r \hat{\psi}_{r}^{(m)}\right)_{r}+\left(2 i s-2 V_{R}-\frac{m^{2}}{r^{2}}\right) \hat{\psi}^{(m)}=0, \quad r \geq R .
$$

This is the Bessel equation for functions of order $m$. Hence, its solution vanishing as $r \rightarrow \infty$ is the $m$-th order Hankel function of the first kind $H_{m}^{(1)}$ :

$$
\hat{\psi}^{(m)}(r, s)=\alpha_{m}(s) H_{m}^{(1)}\left(\sqrt{2 i s-2 V_{R}} r\right), \quad r \geq R,
$$

where $\alpha_{m}(s)$ is an arbitrary multiplier. The radial derivative of $\hat{\psi}^{(m)}$ is computed as

$$
\begin{aligned}
\frac{\partial}{\partial r} \hat{\psi}^{(m)}(r, s) & =\alpha_{m}(s) \sqrt{2 i s-2 V_{R}} H_{m}^{(1)^{\prime}}\left(\sqrt{2 i s-2 V_{R}} r\right) \\
& =\sqrt{2 i s-2 V_{R}} \frac{H_{m}^{(1)^{\prime}}\left(\sqrt{2 i s-2 V_{R}} r\right)}{H_{m}^{(1)}\left(\sqrt{2 i s-2 V_{R}} R\right)} \hat{\psi}^{(m)}(R, s),
\end{aligned}
$$

where we have determined the value of the coefficient $\alpha_{m}(s)$ from (1.6) by setting $r=R$. Finally, the TBCs are obtained by computing the series (1.4), using the inverse Laplace transform and setting $r=R$ :

$$
\begin{aligned}
& \frac{\partial \psi}{\partial r}(R, \theta, t) \\
= & \frac{1}{2 \pi i} \sum_{m \in \mathbb{Z}_{\gamma}} \int_{i \infty}^{\gamma+i \infty} \sqrt{2 i s-2 V_{R}} \frac{H_{m}^{(1)^{\prime}}\left(\sqrt{2 i s-2 V_{R}} R\right)}{H_{m}^{(1)}\left(\sqrt{2 i s-2 V_{R}} R\right)} \hat{\psi}^{(m)}(R, s) e^{s t} d s e^{i m \theta},
\end{aligned}
$$

where $\Gamma=(\gamma-i \infty, \gamma+i \infty)$ is a vertical contour in the complex plane chosen such that all singularities of the integrand are to the left of it.

The TBCs (1.8) are non-local both in time and in space. A strategy to derive a spatially localized version of (1.8) by an asymptotic expansion of the Hankel functions and their derivatives with respect to $s$ can be found in [5].

Because of the nonlocality of the TBCs (1.8), their immediate numerical implementation requires one to store the boundary data $\hat{\psi}^{(m)}(R,$.$) of all the past history$ 
and for all modes $m \in \mathbb{Z}$. Moreover, the discretization of the TBCs (1.8), even in one space dimension, is not trivial at all and has attracted lots of attention. For the many proposed discretization strategies of the TBCs (1.8) in 1D (as well as semi-discrete approaches), we refer the reader to $[1,4,9,11,13,15,21,37,38,43,44,45,46,47,60]$ and references therein. A numerically efficient treatment of the 2D TBCs (1.8) was recently proposed by Jiang and Greengard in [31].

We remark that inadequate discretizations of the TBCs may introduce strong numerical reflections at the boundary or render the discrete initial boundary value problem only conditionally stable; see [22] for a detailed discussion.

REMARK 1.1 (Generalizations of the Schrödinger Equation (1.1a)). The case of a time-dependent exterior potential $V=V_{R}(t)$ can easily be included by the gauge transformation

$$
\varphi=\exp \left(i \int_{0}^{t} V_{R}(t) d s\right) \psi
$$

(cf. [4] or Remark 5 in [6], e.g.) to reduce this case to zero exterior potential. Moreover, some classes of nonlinear Schrödinger equations can be handled by the simple potential strategy of Szeftel [53, 54], i.e. by freezing the potential and updating this nonlinear term at every time step (and recomputing all coefficients!). This approach of Szeftel was transferred to discrete TBCs in [61]. Let us note that a family of absorbing boundary conditions for the 3D case was recently introduced in [27].

Difference equations. We consider a Crank-Nicolson finite difference scheme, which is one of the commonly used discretization methods for the Schrödinger equation. Let us introduce a polar and temporal grid:

$$
\begin{gathered}
r_{-1}<r_{0}<r_{1}<\cdots<r_{J}<\ldots, \quad r_{-1}=-r_{0} ; \quad r_{J-1 / 2}=R \\
r_{j+1 / 2}=\left(r_{j+1}+r_{j}\right) / 2, \Delta r_{j+1 / 2}=r_{j+1}-r_{j}, \Delta r_{j}=r_{j+1 / 2}-r_{j-1 / 2} \\
\theta_{k}=k \Delta \theta, \quad k=0,1, \ldots, K, \quad \Delta \theta=2 \pi / K \\
t_{n}=n \Delta t, \quad n=0,1, \ldots
\end{gathered}
$$

We denote

$$
\psi_{j, k}^{(n)}=\psi\left(r_{j}, \theta_{k}, t_{n}\right), \quad \psi_{j, k}^{(n+1 / 2)}=\left(\psi_{j, k}^{(n+1)}+\psi_{j, k}^{(n)}\right) / 2,
$$

and $\quad V_{j, k}^{(n+1 / 2)}=V\left(r_{j}, \theta_{k}, t_{n+1 / 2}\right)$. Then the Crank-Nicolson scheme reads:

$$
\begin{gathered}
-\frac{2 i}{\Delta t}\left(\psi_{j, k}^{(n+1)}-\psi_{j, k}^{(n)}\right) \\
=\frac{1}{r_{j}} \frac{1}{\Delta r_{j}}\left[\frac{r_{j+1 / 2}\left(\psi_{j+1, k}^{(n+1 / 2)}-\psi_{j, k}^{(n+1 / 2)}\right)}{\Delta r_{j+1 / 2}}-\frac{r_{j-1 / 2}\left(\psi_{j, k}^{(n+1 / 2)}-\psi_{j-1, k}^{(n+1 / 2)}\right)}{\Delta r_{j-1 / 2}}\right] \\
+\frac{1}{r_{j}^{2}} \frac{\psi_{j, k+1}^{(n+1 / 2)}-2 \psi_{j, k}^{(n+1 / 2)}+\psi_{j, k-1}^{(n+1 / 2)}}{\Delta \theta^{2}}-2 V_{j, k}^{(n+1 / 2)} \psi_{j, k}^{(n+1 / 2)}, \\
j=0,1, \ldots ; \quad k=0,1, \ldots, K-1 ; \quad n=0,1, \ldots
\end{gathered}
$$

and the obvious periodic boundary conditions $\psi_{j, 0}^{(n)}=\psi_{j, K}^{(n)}, \psi_{j,-1}^{(n)}=\psi_{j, K-1}^{(n)}$.

REMARK 1.2 (Treatment of singularity at the origin). We use a radial offset grid here such that the coefficient of $\psi_{-1, k}^{(n+1 / 2)}$ is zero. 
The paper is organized as follows. In $\S 2$ we prove the discrete mass conservation property of the Crank-Nicolson scheme and derive discrete TBCs directly for the chosen numerical scheme using the $Z$-transform method. In contrast to the $1 \mathrm{D}$ and the rectangular 2D cases, the convolution coefficients of the discrete TBCs have to be obtained numerically here. Using their large-radius-limit (i.e. the planar problem) as a starting point, they are computed by a recursion from "infinity" back to the finite radius $R$. Next we prove the stability of the recurrence formulas used to obtain the convolution coefficients of the new discrete TBCs for a spatially dependent potential.

In $\S 3$ we discuss the approximation of the convolution coefficients by a discrete sum of exponentials and present an efficient recursion for evaluating these approximate discrete TBCs. Finally, the numerical examples of $\S 4$ illustrate the accuracy, stability, and efficiency of the proposed method.

In the Appendix we briefly revisit discrete TBCs for the 1D Schrödinger equation. We present a new formulation that leads to convolutions coefficients with better decay properties than those from the literature [7, 22].

\section{The discrete TBCs}

First we generate transparent discrete boundary conditions using exact solutions to the difference scheme (1.9) in the exterior domain $r \geq R$.

Reduction to 1D-Problem. In order to reduce the problem to the simpler $1 \mathrm{D}$ case, the discrete Fourier method is used in the $\theta$-direction. Due to the periodic boundary conditions in the angular direction we have

$$
\psi_{j, 0}^{(n)}=\psi_{j, K}^{(n)}, \quad j \in \mathbb{N}_{0}, n \geq 0,
$$

and hence, use the discrete Fourier transform of $\psi_{j, k}^{(n)}$ in the $\theta$-direction:

$$
\psi_{j}^{(m, n)}:=\frac{1}{K} \sum_{k=0}^{K-1} \psi_{j, k}^{(n)} \exp \left(\frac{2 \pi i k m}{K}\right), \quad m=0, \ldots, K-1 .
$$

The scheme (1.9) in the exterior domain $j \geq J-1$ then transforms into:

$$
\begin{gathered}
-\frac{2 i}{\Delta t}\left(\psi_{j}^{(m, n+1)}-\psi_{j}^{(m, n)}\right) \\
=\frac{1}{r_{j}} \frac{1}{\Delta r_{j}}\left[\frac{r_{j+1 / 2}\left(\psi_{j+1}^{(m, n+1 / 2)}-\psi_{j}^{(m, n+1 / 2)}\right)}{\Delta r_{j+1 / 2}}\right. \\
\left.-\frac{r_{j-1 / 2}\left(\psi_{j}^{(m, n+1 / 2)}-\psi_{j-1}^{(m, n+1 / 2)}\right)}{\Delta r_{j-1 / 2}}\right]-2 V_{j}^{(m)} \psi_{j}^{(m, n+1 / 2)}, \\
V_{j}^{(m)}:=V_{R}+\frac{2 \sin ^{2}\left(\frac{\pi m}{K}\right)}{r_{j}^{2} \Delta \theta^{2}}, \quad 0 \leq m \leq K-1, n \geq 0 .
\end{gathered}
$$

The modes $\psi^{(m)}, m=0, \ldots, K-1$ are independent of each other in the exterior domain $r \geq R$ since the potential $V$ is constant there. Therefore we can continue our analysis for each azimuth mode separately.

Thus, by omitting in the sequel the superscript $m$ in the notation, we will consider in the exterior domain $j \geq J-1$ the following discrete 1D-Schrödinger equation:

$$
-i \frac{2 \Delta r_{j} \Delta r_{j+1 / 2}}{\Delta t}\left(\psi_{j}^{(n+1)}-\psi_{j}^{(n)}\right)
$$




$$
\begin{aligned}
=\frac{1}{r_{j}} & {\left[r_{j+1 / 2}\left(\psi_{j+1}^{(n+1 / 2)}-\psi_{j}^{(n+1 / 2)}\right)\right.} \\
& \left.-r_{j-1 / 2} \frac{\Delta r_{j+1 / 2}}{\Delta r_{j-1 / 2}}\left(\psi_{j}^{(n+1 / 2)}-\psi_{j-1}^{(n+1 / 2)}\right)\right]-2 \Delta r_{j} \Delta r_{j+1 / 2} V_{j} \psi_{j}^{(n+1 / 2)},
\end{aligned}
$$

with the spatially dependent potential $V_{j}=V_{R}+2 \sin ^{2}\left(\frac{\pi m}{K}\right) /\left(r_{j}^{2} \Delta \theta^{2}\right)$.

Mass conservation property. There are two important advantages of the second order (in $\Delta r$ and $\Delta t$ ) scheme (2.4): it is unconditionally stable, and it preserves the discrete $L^{2}$-norm in time:

Lemma 2.1. For the scheme (2.4) (considered on $j \in \mathbb{N}_{0}$ ), it holds that

$$
\left\|\psi^{(n)}\right\|_{2}^{2}:=\sum_{j \in \mathbb{N}_{0}} \Delta r_{j}\left|\psi_{j}^{(n)}\right|^{2} r_{j}
$$

is a conserved quantity in time.

Proof. This conservation property can be seen by a discrete energy estimate. First we multiply $(2.4)$ by $\bar{\psi}_{j}^{(n)} r_{j}$ :

$$
\begin{aligned}
& -\frac{2 i}{\Delta t}\left(\psi_{j}^{(n+1)} \bar{\psi}_{j}^{(n)}-\left|\psi_{j}^{(n)}\right|^{2}\right) r_{j} \\
= & \bar{\psi}_{j}^{(n)} D^{0}\left(r_{j} D^{0} \psi_{j}^{(n+1 / 2)}\right)-2 V_{j} \psi_{j}^{(n+1 / 2)} \bar{\psi}_{j}^{(n)} r_{j}, \quad j=0,1, \ldots, \\
& \frac{2 i}{\Delta t}\left(\left|\psi_{j}^{(n+1)}\right|^{2}-\bar{\psi}_{j}^{(n)} \psi_{j}^{(n+1)}\right) r_{j} \\
= & \psi_{j}^{(n+1)} D^{0}\left(r_{j} D^{0} \bar{\psi}_{j}^{(n+1 / 2)}\right)-2 V_{j} \bar{\psi}_{j}^{(n+1 / 2)} \psi_{j}^{(n+1)} r_{j}, \quad j=0,1, \ldots,
\end{aligned}
$$

with the abbreviation of the centered difference quotient

$$
D^{0}=D_{\frac{\Delta r_{j}}{2}}^{0} \text {, i.e. } D^{0} \psi_{j}^{n}=\frac{\psi_{j+1 / 2}^{n}-\psi_{j-1 / 2}^{n}}{\Delta r_{j}} .
$$

Next we subtract (2.6a) from (2.6b)

$$
\begin{aligned}
& \frac{2 i}{\Delta t}\left(\left|\psi_{j}^{(n+1)}\right|^{2}-\left|\psi_{j}^{(n)}\right|^{2}\right) r_{j} \\
= & \psi_{j}^{(n+1)} D^{0}\left(r_{j} D^{0} \bar{\psi}_{j}^{(n+1 / 2)}\right)-\bar{\psi}_{j}^{(n)} D^{0}\left(r_{j} D^{0} \psi_{j}^{(n+1 / 2)}\right) \\
& \quad-V_{j}\left(\left|\psi_{j}^{(n+1)}\right|^{2}-\left|\psi_{j}^{(n)}\right|^{2}\right) r_{j}, \quad j=0,1, \ldots,
\end{aligned}
$$

multiply by $\Delta r_{j}$, sum from $j=0$ to $\infty$, and apply summation by parts:

$$
\begin{aligned}
& \frac{2 i}{\Delta t} \sum_{j=0}^{\infty}\left(\left|\psi_{j}^{(n+1)}\right|^{2}-\left|\psi_{j}^{(n)}\right|^{2}\right) r_{j} \Delta r_{j} \\
= & -\sum_{j \in \mathbb{N}_{0}+\frac{1}{2}}\left(D^{0} \bar{\psi}_{j}^{(n+1 / 2)}\right)\left(D^{0} \psi_{j}^{(n+1)}\right) r_{j} \Delta r_{j}-\left(D^{0} \bar{\psi}_{-\frac{1}{2}}^{(n+1 / 2)}\right) \psi_{0}^{(n+1)} r_{-\frac{1}{2}}
\end{aligned}
$$




$$
\begin{aligned}
& +\sum_{j \in \mathbb{N}_{0}+\frac{1}{2}}\left(D^{0} \psi_{j}^{(n+1 / 2)}\right)\left(D^{0} \bar{\psi}_{j}^{(n)}\right) r_{j} \Delta r_{j}+\left(D^{0} \psi_{-\frac{1}{2}}^{(n+1 / 2)}\right) \bar{\psi}_{0}^{(n)} r_{-\frac{1}{2}} \\
& -\sum_{j=0}^{\infty} V_{j}\left(\left|\psi_{j}^{(n+1)}\right|^{2}-\left|\psi_{j}^{(n)}\right|^{2}\right) r_{j} \Delta r_{j} .
\end{aligned}
$$

Now, the boundary terms in (2.7) vanish since $r_{-\frac{1}{2}}=0$, and hence

$$
\begin{aligned}
\frac{2 i}{\Delta t} \sum_{j=0}^{\infty}\left(\left|\psi_{j}^{(n+1)}\right|^{2}-\left|\psi_{j}^{(n)}\right|^{2}\right) r_{j} \Delta r_{j}= & -\frac{1}{2} \sum_{j \in \mathbb{N}_{0}+\frac{1}{2}}\left(\left|D^{0} \bar{\psi}_{j}^{(n+1)}\right|^{2}-\left.D^{0} \bar{\psi}_{j}^{(n)}\right|^{2}\right) r_{j} \Delta r_{j} \\
& -\sum_{j=0}^{\infty} V_{j}\left(\left|\psi_{j}^{(n+1)}\right|^{2}-\left|\psi_{j}^{(n)}\right|^{2}\right) r_{j} \Delta r_{j}
\end{aligned}
$$

Finally, taking imaginary parts one obtains the desired result.

Discrete TBCs for a single azimuth mode. Discrete transparent boundary conditions for the 1D Schrödinger equation with constant coefficients of the difference scheme in the exterior domain were introduced by Arnold [7] (cf. the Appendix for an improved variant, which is the bases of our presentation below). Here we derive discrete TBCs for the scheme (2.4) with spatially varying coefficients. Analogous to the continuous case of $\S 1$, the idea is to eliminate the exterior problem $j>J$ and to replace it by a discrete $\mathrm{DtN}$ map.

We use the $Z$-transform of the sequence $\left\{\psi_{j}^{(n)}\right\}, n \in \mathbb{N}_{0}$ (with $j$ considered fixed) which is defined as the Laurent series (see [20])

$$
\mathcal{Z}\left\{\psi_{j}^{(n)}\right\}=\hat{\psi}_{j}(z):=\sum_{n=0}^{\infty} \psi_{j}^{(n)} z^{-n}, \quad z \in \mathbb{C}, \quad|z|>R_{\hat{\psi}_{j}},
$$

and $R_{\hat{\psi}_{j}}$ denotes the convergence radius of the series. Now the transformed exterior scheme (2.4) reads

$$
\begin{aligned}
& -i \rho_{j} \frac{z-1}{z+1} \hat{\psi}_{j}(z) \\
= & \frac{1}{r_{j}}\left[r_{j+1 / 2}\left(\hat{\psi}_{j+1}(z)-\hat{\psi}_{j}(z)\right)-r_{j-1 / 2} \frac{\Delta r_{j+1 / 2}}{\Delta r_{j-1 / 2}}\left(\hat{\psi}_{j}(z)-\hat{\psi}_{j-1}(z)\right)\right] \\
& \quad-2 \Delta r_{j} \Delta r_{j+1 / 2} V_{j} \hat{\psi}_{j}(z), \quad j \geq J-1,
\end{aligned}
$$

with the mesh ratio $\rho_{j}=4 \Delta r_{j} \Delta r_{j+1 / 2} / \Delta t$ and the spatially dependent potential $V_{j}=$ $V_{R}+2 \sin ^{2}\left(\frac{\pi m}{K}\right) /\left(r_{j}^{2} \Delta \theta^{2}\right)$. Note that we used here the following assumption on $\psi^{0}$ :

$$
\psi_{j}^{0}=0, \quad j \geq J-2 .
$$

Thus we obtain a homogeneous second order difference equation with varying coefficients of the form

$$
a_{j} \hat{\psi}_{j+1}(z)+b_{j}(z) \hat{\psi}_{j}(z)+c_{j} \hat{\psi}_{j-1}(z)=0, \quad j \geq J-1,
$$

where

$$
a_{j}=\frac{r_{j+1 / 2}}{r_{j}}
$$




$$
\begin{aligned}
b_{j}(z) & =-\frac{1}{r_{j}}\left[r_{j+1 / 2}+r_{j-1 / 2} \frac{\Delta r_{j+1 / 2}}{\Delta r_{j-1 / 2}}\right]+i \rho_{j} \frac{z-1}{z+1}-2 \Delta r_{j} \Delta r_{j+1 / 2} V_{j}, \\
c_{j} & =\frac{r_{j-1 / 2}}{r_{j}} \frac{\Delta r_{j+1 / 2}}{\Delta r_{j-1 / 2}} .
\end{aligned}
$$

REMARK 2.2 (Uniform offset grid). In the special case of a uniform radial offset grid $r_{j}=\left(j+\frac{1}{2}\right) \Delta r, j \geq J-1$, we obtain

$$
\begin{aligned}
a_{j} & =\frac{j+1}{j+\frac{1}{2}}, \quad c_{j}=\frac{j}{j+\frac{1}{2}}, \\
b_{j}(z) & =-2+i \rho \frac{z-1}{z+1}-2 \Delta r^{2} V_{R}-4 \frac{\sin ^{2}\left(\frac{\pi m}{K}\right)}{(j+1 / 2)^{2} \Delta \theta^{2}} .
\end{aligned}
$$

But such a uniform grid is not a requirement for the rest of this section.

For the formulation of the $Z$-transformed discrete TBCs at $j=J$ we regard the ratio $\hat{\ell}_{j}(z)$ of the decaying (as $j \rightarrow \infty$ ) fundamental solution to (2.10) at two adjacent points:

$$
\hat{\ell}_{j}(z)=\frac{\hat{\psi}_{j}(z)}{\hat{\psi}_{j-1}(z)}, \quad j \geq J,
$$

and get from (2.12) the following Riccati difference equation (cf. §1.6 in [33]) with variable coefficients:

$$
\hat{\ell}_{j}(z)\left(a_{j} \hat{\ell}_{j+1}(z)+b_{j}(z)\right)+c_{j}=0, \quad j \geq J
$$

Suppose (for the moment) that the coefficients $\hat{\ell}_{j}(z)$ are known. Setting $j=J+1$ we get from (2.15)

$$
\hat{\ell}_{J+1}(z)=\frac{\hat{\psi}_{J+1}(z)}{\hat{\psi}_{J}(z)} .
$$

Calculating the inverse $Z$-transformation we obtain the discrete convolution

$$
\psi_{J+1}^{(n)}=\ell_{J+1}^{(n)} * \psi_{J}^{(n)} .
$$

Hence, our discrete TBC reads explicitly

$$
\psi_{J+1}^{(n)}-\ell_{J+1}^{(0)} \psi_{J}^{(n)}=\sum_{p=1}^{n-1} \ell_{J+1}^{(n-p)} \psi_{J}^{(p)},
$$

when using the assumption (1.2)

$$
\psi_{j}^{(0)}=0, \quad j \geq J
$$

Asymptotic behavior of the convolution coefficients. We shall now discuss the behavior of the coefficients $\ell_{J+1}^{(n)}$ for $n \rightarrow \infty$, which will allow us to find an alternative, numerically more suitable formulation of the discrete TBC (2.19). First we remark that this discrete TBC is structurally different from those introduced in 
the literature $[7,8,22,49]$. There, the discrete convolution is always placed at the outer of the two boundary grid points. But in (2.18) it is implemented at the inner boundary point $j=J$. This small modification yields a very different behavior of the convolution coefficients $\ell^{(n)}$ : Here, the coefficients $\ell^{(n)}$ decay like $\mathcal{O}\left(n^{-3 / 2}\right)$ (cf. the Appendix for details). But they are oscillatory in [7, 8, 22, 49]. Hence, it is necessary there to instead use a linear combination of $\ell_{j}^{(n)}$ and $\ell_{j}^{(n-1)}$, which is much "smoother" (considered as functions of $n$ ).

While the asymptotic behavior of $\ell^{(n)}$ (for $n$ large) was determined from explicit formulas in $[22,49]$, this is unfeasible here. It can, however, be deduced from the singularities of $\hat{\ell}_{j}(z), z \in \mathbb{C}$. First we consider (2.12) in the limiting case of a large radial index $j$. Here and in the sequel we make the assumption of an asymptotically equidistant grid (i.e. $\Delta r_{j} \rightarrow \Delta r$ ). (2.12) then reads

$$
\hat{\psi}_{j+1}(z)+\left(-2+i \rho \frac{z-1}{z+1}-2 \Delta r^{2} V_{R}\right) \hat{\psi}_{j}(z)+\hat{\psi}_{j-1}(z)=0,
$$

with $\rho=4 \Delta r^{2} / \Delta t$. This finite difference equation with constant coefficients has solutions of power law form $\hat{\psi}_{j}(z)=c\left(\hat{\ell}_{\infty}(z)\right)^{j}$. Using this ansatz in (2.21) yields the decaying solution as $j \rightarrow \infty$ (i.e. $\left|\hat{\ell}_{\infty}(z)\right|<1$ ) with

$$
\begin{aligned}
\hat{\ell}_{\infty}(z)= & -\frac{i \rho}{2} \frac{z-1}{z+1}+\Delta r^{2} V_{R} \\
& -\frac{1}{z+1} \sqrt{\left[(z+1)\left(1+\Delta r^{2} V_{R}\right)-\frac{i \rho}{2}(z-1)\right]^{2}-(z+1)^{2}} .
\end{aligned}
$$

Note that this formula coincides with the 1D case and the planar 2D case (cf. the Appendix or $[22,10])$. This is no surprise since the curvature of large circles tends to zero for $R \rightarrow \infty$.

In (2.22) the branch of the square root has to be chosen such that $\left|\hat{\ell}_{\infty}(z)\right| \leq 1$ holds for $z \geq 1$ which selects the decaying solution $\hat{\psi}_{j}(z)$. $\hat{\ell}_{\infty}$ has no pole at $z=-1$, but two branch points on the complex unit circle, due to the quadratic polynomial under the square root. For the special case $V_{R}=0$ that we shall illustrate numerically, they are located at $z_{1}=1, z_{2}=(\rho-4 i) /(\rho+4 i)$. These branch points manifest themselves as kinks of $\operatorname{Im} \hat{\ell}_{\infty}(z)$, for $z$ on the unit circle.

Next we discuss the singularities of $\hat{\ell}_{j}(z)$ for $j$ finite. Subsequent $\hat{\ell}_{j}(z)$ 's are related by the recursion (2.16). But since $b_{j}(z)$ is real on the unit circle (as well as $\left.a_{j}, c_{j}\right)$, the kinks of $\operatorname{Im} \hat{\ell}_{j}(z)$ are still located at $z_{1}=1, z_{2}=(\rho-4 i) /(\rho+4 i)$ for all $j$ and for all modes $m$.

Now we turn to a discussion of the asymptotic behavior of $\ell_{J}^{(n)}$ for $n \rightarrow \infty$. Since $\ell_{J}^{(n)}$ are just the Fourier coefficients of the $2 \pi$-periodic function $\hat{\ell}_{J}\left(e^{i \varphi}\right)$ (cf. (2.9)), its asymptotic behavior is determined by the singularities of $\hat{\ell}_{J}(z)$ on the unit circle. Hence, the two square root singularities, mentioned above, imply

$$
\ell_{J}^{(n)} \sim\left(c_{1} z_{1}^{n}+c_{2} z_{2}^{n}\right) n^{-3 / 2} .
$$

To compensate the oscillations with the higher frequency (determined by $z_{2}^{n}$ ) we define the following summed convolution coefficients:

$$
\begin{aligned}
& s^{(0)}:=\ell_{J+1}^{(0)}, \\
& s^{(n)}:=\ell_{J+1}^{(n)}-z_{2} \ell_{J+1}^{(n-1)}, \quad n \geq 1 .
\end{aligned}
$$


This strategy is different from $[22,10,49]$ since the asymptotic behavior of the coefficients $\ell^{(n)}$ is very different in both cases. These new coefficients $s^{(n)}$ are now less oscillatory than the $\ell_{J+1}^{(n)}$. Hence, they are a better starting point for computing approximate convolution coefficients (see $\S 3$ below). The discrete $T B C$ for a single azimuth mode now reads

$$
\psi_{J+1}^{(n)}-s^{(0)} \psi_{J}^{(n)}=\sum_{p=1}^{n-1} s^{(n-p)} \psi_{J}^{(p)}+z_{2} \psi_{J+1}^{(n-1)} .
$$

Calculation of convolution coefficients. In order to find a solution to (2.16) we use the method of series. Let us consider the Laurent series for $\hat{\ell}_{j}(z)$ :

$$
\hat{\ell}_{j}(z)=\ell_{j}^{(0)}+\ell_{j}^{(1)} z^{-1}+\cdots+\ell_{j}^{(n)} z^{-n}+\ldots, \quad|z| \geq 1 .
$$

We define the auxiliary functions

$$
\begin{aligned}
\alpha_{j}(z) & :=\frac{b_{j}(z)}{a_{j}}, \\
\alpha_{j} & :=\lim _{z \rightarrow \infty} \alpha_{j}(z), \\
\beta_{j} & :=\frac{c_{j}}{a_{j}} .
\end{aligned}
$$

Then (2.16) reads

$$
\hat{\ell}_{j}(z)\left(\hat{\ell}_{j+1}(z)+\alpha_{j}(z)\right)+\beta_{j}=0, \quad j \geq J .
$$

Substituting (2.26) for (2.28) we get

$$
\begin{aligned}
\left(\ell_{j}^{(0)}+\ell_{j}^{(1)} z^{-1}+\right. & \left.\cdots+\ell_{j}^{(n)} z^{-n}+\ldots\right) \\
& \times\left(\left(\ell_{j+1}^{(0)}+\ell_{j+1}^{(1)} z^{-1}+\cdots+\ell_{j+1}^{(n)} z^{-n}+\ldots\right)+\alpha_{j}(z)\right)+\beta_{j}=0 .
\end{aligned}
$$

We shall now discuss the computation of the coefficients $\ell_{j}^{(n)}$ for individual indices $n$ :

Coefficient $\boldsymbol{\ell}_{\mathbf{j}}^{(\mathbf{0})}$. Taking $|z| \rightarrow \infty$ we have the following recurrence equation for $\ell_{j}^{(0)}$ :

$$
\ell_{j}^{(0)}\left(\ell_{j+1}^{(0)}+\alpha_{j}\right)+\beta_{j}=0 .
$$

We shall solve this equation by "iteration from infinity", i.e. starting from a (large) index $J_{\infty}$, putting an initial value $\ell_{J_{\infty}}^{(0)}:=\ell_{\infty}^{(0)}$, and running the recursion from $J_{\infty}$ to $J+1$ :

$$
\ell_{j}^{(0)}=\frac{-\beta_{j}}{\ell_{j+1}^{(0)}+\alpha_{j}}, \quad j=J_{\infty}-1, J_{\infty}-2, \ldots, J+1 .
$$

Note that a very large index $J_{\infty}$ corresponds to a very large radius $r_{J_{\infty}}$. Therefore we can use the coefficient $\ell^{(0)}$ from the $1 \mathrm{D}$ case (cf. (A.4), or the $2 \mathrm{D}$ plane case) as the starting value $\ell_{\infty}^{(0)}$. It is obtained from $(2.22)$ as $\ell_{\infty}^{(0)}=\hat{\ell}_{\infty}(z=\infty)$.

ThEOREM 2.3 (Stability of the recurrence relation). Let $\left|\alpha_{j}\right| \geq 2 \frac{j+1 / 2}{j+1}, \beta_{j} \leq \frac{j}{j+1}$, and $\left|\ell_{J_{\infty}}^{(0)}\right|<\beta_{J_{\infty}}$. Then: 
a) $\left|\ell_{j}^{(0)}\right|<\beta_{j} \leq \frac{j}{j+1}<1 ;$ and

b) the recurrence formula (2.31) is stable with respect to small perturbations.

Proof. Part (a) is proved by induction. Suppose $\left|\ell_{j+1}^{(0)}\right|<\beta_{j+1} \leq \frac{j}{j+1}$. Hence

$$
\left|\ell_{j+1}^{(0)}+\alpha_{j}\right| \geq\left|\alpha_{j}\right|-\left|\ell_{j+1}^{(0)}\right|>2 \frac{j+1 / 2}{j+1}-\frac{j}{j+1}=1 .
$$

Therefore

$$
\left|\ell_{j}^{(0)}\right|=\frac{\beta_{j}}{\left|\ell_{j+1}^{(0)}+\alpha_{j}\right|}<\beta_{j} .
$$

To prove (b) and establish the stability we suppose that we have a perturbation $\ell_{j+1}^{(0)}+\delta_{j+1}$ instead of $\ell_{j+1}^{(0)}$ with $\left|\delta_{j+1}\right|<1$. Let us consider the evolution of $\delta_{j}$ by comparing (2.31) with

$$
\ell_{j}^{(0)}+\delta_{j}=\frac{-\beta_{j}}{\ell_{j+1}^{(0)}+\delta_{j+1}+\alpha_{j}}, \quad j=J_{\infty}-1, J_{\infty}-2, \ldots, J+1 .
$$

Evidently we obtain:

$$
\begin{aligned}
\delta_{j} & =\frac{-\beta_{j}}{\ell_{j+1}^{(0)}+\delta_{j+1}+\alpha_{j}}-\frac{-\beta_{j}}{\ell_{j+1}^{(0)}+\alpha_{j}}=\delta_{j+1} \frac{-\ell_{j}^{(0)}}{\ell_{j+1}^{(0)}+\delta_{j+1}+\alpha_{j}} \\
& =\delta_{j+1} \frac{-\ell_{j}^{(0)}}{-\beta_{j} / \ell_{j}^{(0)}+\delta_{j+1}} .
\end{aligned}
$$

Therefore we get

$$
\begin{aligned}
\left|\delta_{j}\right| & =\left|\delta_{j+1}\right| \frac{\left|\ell_{j}^{(0)}\right|}{\left|\beta_{j} / \ell_{j}^{(0)}-\delta_{j+1}\right|} \\
& \leq\left|\delta_{j+1}\right| \frac{\left|\ell_{j}^{(0)}\right|^{2}}{\beta_{j}-\left|\ell_{j}^{(0)}\right|\left|\delta_{j+1}\right|}<\left|\delta_{j+1}\right| \frac{\left|\ell_{j}^{(0)}\right|^{2}}{\beta_{j}} \frac{1}{1-\left|\delta_{j+1}\right|},
\end{aligned}
$$

and hence

$$
\frac{\left|\delta_{j}\right|}{\left|\delta_{j+1}\right|} \sim \frac{\left|\ell_{j}^{(0)}\right|^{2}}{\beta_{j}}<\beta_{j}<1,
$$

for $\left|\delta_{j+1}\right| \ll 1$. Thus the recursion (2.31) is stable with respect to small perturbations (e.g. for truncation errors or for an "incorrect" initial guess $\left.\ell_{J_{\infty}}^{(0)}:=\ell_{\infty}^{(0)}\right)$.

REMARK 2.4. The assumptions $\left|\alpha_{j}\right| \geq 2 \frac{j+1 / 2}{j+1}$ and $\beta_{j} \leq \frac{j}{j+1}$ in the previous theorem are valid for the definitions (2.14) (i.e. for a uniform offset grid) and $V_{R} \geq 0$. Moreover $\left|\ell_{J_{\infty}}\right|<\beta_{J_{\infty}}$ holds true for $J_{\infty}$ large enough, since $\beta_{j} \stackrel{j \rightarrow \infty}{\longrightarrow} 1$ for an asymptotically equidistant grid.

REMARK 2.5. The estimate (2.33) explains the fast convergence of the recursion (2.31) to the correct value $\ell_{J}^{(0)}$ in spite of taking an "incorrect" initial guess $\ell_{J_{\infty}}^{(0)}:=\ell_{\infty}^{(0)}$; see 
the numerical examples below. Indeed, due to (2.33) we can hope for an exponential decay of $\left|\delta_{j}\right|$ with the factor $\left|\ell_{j}^{(0)}\right|^{2} / \beta_{j} \sim\left|\ell_{j}^{(0)}\right|$. For instance, the value $\left|\ell_{j}^{(0)}\right|$ is estimated from the case of the "frozen" coefficients at $J_{\infty}$ :

$$
\left|\ell_{j}^{(0)}\right| \sim\left|\ell_{\infty}^{(0)}\right|<1
$$

where $\ell_{\infty}^{(0)}=\hat{\ell}_{\infty}(z=\infty)$ from $(2.22)$. $(2.13)$ :

Coefficient $\ell_{\mathbf{j}}^{(\mathbf{1})}$. Now we consider the calculation of $\ell_{j}^{(1)}$. We have from (2.27),

$$
\alpha_{j}(z):=\alpha_{j}-\gamma_{j}\left(z^{-1}-z^{-2}+z^{-3}-\ldots\right)
$$

with $\gamma_{j}:=\alpha_{j}-\bar{\alpha}_{j}$. From (2.29) and (2.34) we can write

$$
\begin{aligned}
\left(\ell_{j}^{(0)}+\ell_{j}^{(1)}\right. & \left.z^{-1}+\mathrm{O}\left(z^{-2}\right)\right) \\
& \times\left(\left(\ell_{j+1}^{(0)}+\ell_{j+1}^{(1)} z^{-1}+\mathrm{O}\left(z^{-2}\right)\right)+\left(\alpha_{j}-\gamma_{j} z^{-1}+\mathrm{O}\left(z^{-2}\right)\right)\right)+\beta_{j}=0 .
\end{aligned}
$$

Annihilating leading order terms by using (2.30) we collect terms of order $z^{-1}$ :

$$
\ell_{j}^{(0)} \ell_{j+1}^{(1)}-\ell_{j}^{(0)} \gamma_{j}+\ell_{j}^{(1)} \ell_{j+1}^{(0)}+\ell_{j}^{(1)} \alpha_{j}=0 .
$$

Therefore the recursion is defined by

$$
\ell_{j}^{(1)}=-\frac{\ell_{j}^{(0)} \ell_{j+1}^{(1)}-\ell_{j}^{(0)} \gamma_{j}}{\ell_{j+1}^{(0)}+\alpha_{j}}, \quad j=J_{\infty}-1, J_{\infty}-2, \ldots, J+1,
$$

with an initial value $\ell_{J_{\infty}}^{(1)}:=\ell_{\infty}^{(1)}$.

Coefficient $\ell_{\mathbf{j}}^{(\mathbf{n})}$. The case of $\ell_{j}^{(n)}$ with $n \geq 2$ is considered similarly by truncating terms of $\mathrm{O}\left(z^{-n-1}\right)$ in (2.35). We get the following recursion formula:

$$
\ell_{j}^{(n)}=-\frac{\sum_{k=0}^{n-1} \ell_{j}^{(k)}\left[\ell_{j+1}^{(n-k)}+\gamma_{j}(-1)^{n-k}\right]}{\ell_{j+1}^{(0)}+\alpha_{j}}, \quad j=J_{\infty}-1, J_{\infty}-2, \ldots, J+1,
$$

with an initial value $\ell_{J_{\infty}}^{(n)}:=\ell_{\infty}^{(n)}$ that can be taken from the $1 \mathrm{D}$ plane case: $\ell_{J_{\infty}}^{(n)} \equiv \ell_{\infty}^{(n)}$. Notice that (2.37) is a particular case of (2.38) for $n=1$.

THEOREM 2.6. Under the conditions of Theorem 2.3 the recurrence formula (2.38) is stable with respect to small perturbations.

Proof. Let us write (2.38) as an iteration with respect to the index $n$ :

$$
\ell_{j}^{(n)}=\frac{\ell_{j}^{(0)}}{\ell_{j+1}^{(0)}+\alpha_{j}} \ell_{j+1}^{(n)}+F\left(\left\{\ell_{j}^{\left(n_{1}<n\right)}\right\},\left\{\ell_{j+1}^{\left(n_{1}<n\right)}\right\}\right)
$$

$j=J_{\infty}-1, J_{\infty}-2, \ldots, J+1$, where the function $F$ contains the remaining terms with indexes $n_{1}<n$. Suppose that the coefficients $\left\{\ell_{j}^{\left(n_{1}\right)}\right\}, n_{1}=0,1, \ldots, n-1, j=J_{\infty}-$ $1, J_{\infty}-2, \ldots, J+1$ are exact (or known with good accuracy). Then the stability of (2.38) is determined by the magnitude of the multiplier

$$
\frac{\ell_{j}^{(0)}}{\ell_{j+1}^{(0)}+\alpha_{j}}
$$


We have from (2.32):

$$
\frac{\left|\ell_{j}^{(0)}\right|}{\left|\ell_{j+1}^{(0)}+\alpha_{j}\right|}<\frac{\beta_{j}}{\left|\ell_{j+1}^{(0)}+\alpha_{j}\right|}<\beta_{j} \leq \frac{j}{j+1}<1 .
$$

REMARK 2.7. The proof of Theorem 2.6 is made by induction with respect to $n=$ $1,2, \ldots$ under the assumption that the previous coefficients for $n_{1}<n$ are (almost) correct. In practice, while calculating the coefficients $\ell_{j}^{(n)}$ we must fix some value $J_{\infty}$ and take an "incorrect" initial value $\ell_{J_{\infty}}^{(n)}:=\ell_{\infty}^{(n)}$. This could give a numerical instability. However, $\ell_{j}^{(0)}$ converges sufficiently fast to its correct value and reaches a very good approximation after, say, $J_{0}$ steps of the recursion (2.31). Hence, we can start the recursion for $\ell_{j}^{(1)}$ a little bit "later", i.e. with the delayed initial index $j=J_{\infty}-J_{0}$. Similarly for $\ell_{j}^{(2)}$, the initial index can be chosen as $j=J_{\infty}-2 J_{0}$, etc. In our numerical tests practical values for $J_{0}$ satisfy $0 \leq J_{0} \leq 5$.

Sample calculations of the coefficients $\ell_{\mathbf{j}}^{(\mathbf{n})}$. We demonstrate the efficiency of the proposed algorithm for the following example. For the radius we consider $R=1$ and we discretize the circular domain $[0, R] \times[0,2 \pi]$ with the uniform step sizes $\Delta r=1 / 200$ and $\Delta \theta=2 \pi / 200$. For the time step size we take $\Delta t=0.0003$ and calculate the convolution coefficients $\ell_{j}^{(n)}$ with (2.38) for the free Schrödinger equation (i.e. $V=0)$ for $n=0, \ldots, 60$. In a first set of calculations we run the algorithm with the choice $J_{\infty}=550$ (which corresponds to $r=3.75$ ) and a retarding shift $J_{0}=5$. Here we just discuss the results for the mode $m=1$, but all other modes behave similarly. In Figure 2.1 we show the absolute values of the last seven coefficients $\ell_{j}^{(54)}, \ldots, \ell_{j}^{(60)}$ as a function of $r \in[R, 3.75]$. We observe a good convergence of the coefficients while approaching the artificial boundary $R=1$ from the exterior domain. An error estimate is provided by a second calculation, where we compute convolution coefficients $\tilde{\ell}_{j}^{(n)}$ with $J_{\infty}=1100$ and the same discretization parameters as before. The difference $\left|\ell_{j}^{(n)}-\tilde{\ell}_{j}^{(n)}\right|$ is plotted for $n=54, \ldots, 60$ in Figure 2.2. With values of the order $\mathcal{O}\left(10^{-14}\right)$ near the artificial boundary this error is about the rounding error of Matlab. The influence of the retarding shift parameter $J_{0}$ can be seen by comparing Figure 2.1 with Figure 2.3. In the third run we determine the convolution coefficients (still with the same discretization parameters and $J_{\infty}=550$ ) but with $J_{0}=3$. The absolute values of these convolution coefficients are presented in Figure 2.3. The oscillations in $\ell_{j}^{(n)}$ due to the instability near $J_{\infty}$ in this plot are more obvious than in the coefficients computed with $J_{0}=5$ shown in Figure 2.1. But also for the choice $J_{0}=3$ the coefficients converge well while approaching $r=R$.

2D discrete TBC. In the Fourier transformed space, i.e. in terms of separate azimuthal modes, the discrete TBCs read (this is Equation (2.25) with recovered mode index $m$ ):

$$
\psi_{J+1}^{(m, n)}-s_{m}^{(0)} \psi_{J}^{(m, n)}=\sum_{p=1}^{n-1} s_{m}^{(n-p)} \psi_{J}^{(m, p)}+z_{2} \psi_{J+1}^{(m, n-1)},
$$

where $m=0, \ldots, K-1, n \geq 1$. Note that (2.3) implies the following symmetry in the convolution coefficients: $s_{m}^{(n)}=s_{K-m}^{(n)}$. In order to obtain the discrete TBC in the 


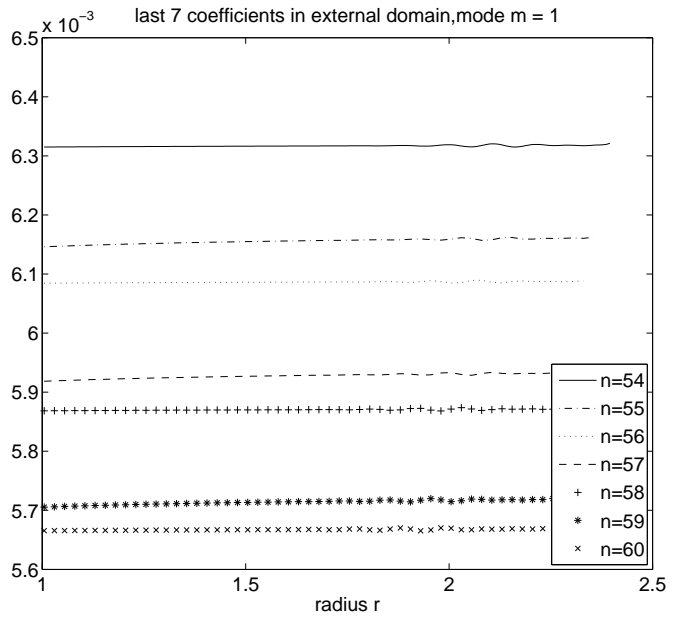

FIG. 2.1. Absolute values of last seven coefficients $\ell_{j}^{(n)}, n=54, \ldots, 60, J_{\infty}=550, J_{0}=5 ; m=1$.

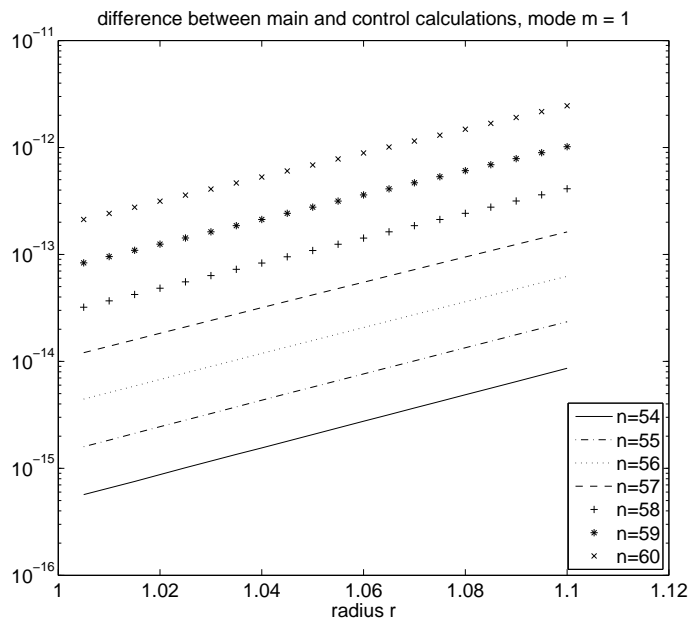

FIG. 2.2. Absolute values of difference $\left|\ell_{j}^{(n)}-\tilde{\ell}_{j}^{(n)}\right|$ of last seven coefficients calculated with $J_{\infty}=550$ and $J_{\infty}=1100 ; m=1$.

physical space let us introduce the diagonal $K \times K$ matrices

$$
\mathbf{s}^{(p)}=\operatorname{diag}\left\{s_{m}^{(p)}\right\}, \quad m=0, \ldots, K-1 ; p=0,1,2, \ldots
$$

We shall also use the matrices $\mathbf{F}$ and $\mathbf{F}^{-\mathbf{1}}$ of, respectively, the direct and inverse Fourier transform in the $\theta$-direction acting in accordance with (2.2):

$$
\hat{\psi}_{j}^{(n)}=\mathbf{F} \tilde{\psi}_{j}^{(n)},
$$

with the vectors

$$
\hat{\psi}_{j}^{(n)}=\left\{\psi_{j}^{(m, n)}\right\}_{m=0}^{K-1}, \quad \tilde{\psi}_{j}^{(n)}=\left\{\psi_{j, k}^{(n)}\right\}_{k=0}^{K-1}
$$




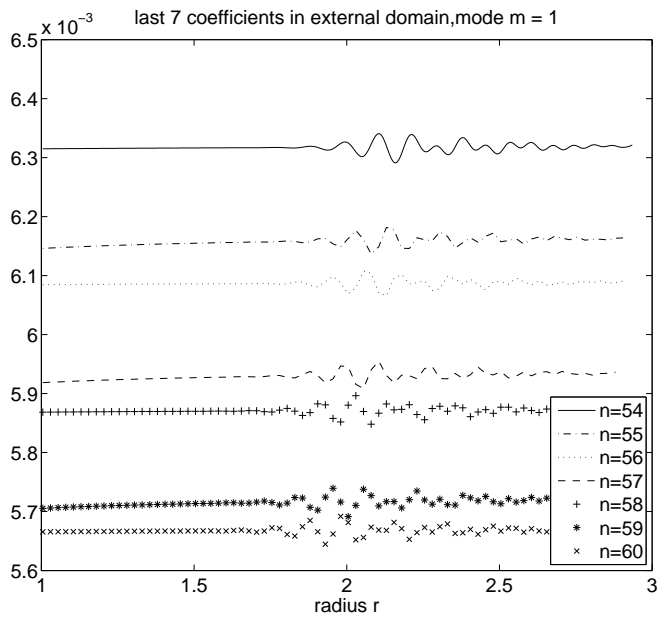

FIG. 2.3. Absolute values of last seven coefficients, $J_{\infty}=550, J_{0}=3 ; m=1$.

Then, multiplying (2.40) by $\mathbf{F}^{-\mathbf{1}}$ we get the following $2 D$ discrete $T B C$ :

$$
\tilde{\psi}_{J+1}^{(n)}-\mathbf{F}^{-1} \mathbf{s}^{(0)} \mathbf{F} \tilde{\psi}_{J}^{(n)}=\mathbf{F}^{-1} \sum_{p=1}^{n-1} \mathbf{s}^{(n-p)} \mathbf{F} \tilde{\psi}_{J}^{(p)}+z_{2} \tilde{\psi}_{J+1}^{(n-1)} .
$$

Here, we choose to formulate the discrete TBC (2.41) at the boundary of the computational interval and one grid point in the exterior domain. In accordance with (2.20) we have assumed that the initial condition satisfies $\psi_{j, k}^{(0)}=0, j \geq J ; k=0, \ldots, K-1$.

Using the formula (2.41) for calculations permits us to avoid any boundary reflections and it renders the fully discrete scheme unconditionally stable (just like the underlying Crank-Nicolson scheme). Note that we need to evaluate for each mode $m$ just one convolution of (2.41) at each time step (at the endpoint of the interval $\left.\left[0, t_{n}\right]\right)$. Since the other points of this convolution are not needed, using an FFT is not practical.

REMARK 2.8 (Computational effort for 2D discrete TBC). The computational costs of 2D discrete TBCs for constant potentials consist of two parts: First, the computation of the convolution coefficients via the recursion from infinity (2.31), (2.37), (2.38) has to be performed only once for each potential and it amounts to $\mathrm{O}\left(\left(J_{\infty}-J\right) K N^{2}\right)$ for the exact DTBCs and $\mathrm{O}\left(\left(J_{\infty}-J\right) K L^{2}\right)+\mathrm{O}(K L N)$ with the approximation (3.1). Secondly, the evaluation of the TBC (2.41) during the solution of the Schrödinger equation incurs costs of $\mathrm{O}\left(K N^{2}\right)+\mathrm{O}(N K \log K)$ without approximation and $\mathrm{O}(K L N)+\mathrm{O}(N K \log K)$ with the sum-of-exponentials approximation, cf. $\S 3$.

\section{Approximation by sums of exponentials}

An ad-hoc implementation of the discrete convolution

$$
\sum_{p=1}^{n-1} s^{(n-p)} \psi_{J}^{(p)}
$$


in (2.25) with convolution coefficients $s^{(n)}$ from (2.24) has still one disadvantage. The boundary conditions are non-local both in time and space and therefore computations are too expensive. As a remedy, to get rid of the time non-locality, we proposed already in [10] the sum of exponentials ansatz, i.e. to approximate the convolution coefficients (2.24) by a finite sum (say $L$ terms) of exponentials that decay with respect to time. This approach allows for a fast (approximate) evaluation of the discrete convolution (2.25) since the convolution can now be evaluated with a simple recurrence formula for $L$ auxiliary terms and the numerical effort per time step now stays constant.

Let us note that similar tricks have been proposed in [24] for the heat equation, in [50] for the continuous TBC of the 3D wave equation, in [31] for the TBC of the 2D Schrödinger equation, and developed in $[2,19,25,51,52]$ for various hyperbolic problems.

In the sequel we will briefly review this ansatz [10]. In order to derive a fast numerical method to calculate the discrete convolutions in (2.40), we approximate the coefficients $s^{(n)}$ by the following ansatz (sum of exponentials):

$$
s^{(n)} \approx \tilde{s}^{(n)}:= \begin{cases}s^{(n)}, & n=0,1, \ldots, \nu-1, \\ \sum_{l=1}^{L} b_{l} q_{l}^{-n}, & n=\nu, \nu+1, \ldots,\end{cases}
$$

where $L, \nu \in \mathbb{N}$ are a fixed numbers. Evidently, the approximation properties of $\tilde{s}^{(n)}$ depend on $L, \nu$, and the corresponding set $\left\{b_{l}, q_{l}\right\}$. Thus, the choice of an (in some sense) optimal approximation of this type is a difficult nonlinear problem. Below we propose a deterministic method of finding $\left\{b_{l}, q_{l}\right\}$ for fixed $L$ and $\nu$.

REMARK 3.1. The "split" definition of $\left\{\tilde{s}^{(n)}\right\}$ in (3.1) is motivated by the fact that the implementation of the discrete TBCs (2.40) involves a convolution sum with $p$ ranging only from 1 to $p=n-1$. Since the first coefficient $s^{(0)}$ does not appear in this convolution, it makes no sense to include it in our sum of exponential approximation, which aims at simplifying the evaluation of the convolution. Hence, one may choose $\nu=1$ in (3.1). The "special form" of $\ell_{\infty}^{(0)}$ and $\ell_{\infty}^{(1)}$ given in [10] suggests even to exclude $s^{(1)}$ from this approximation and to choose $\nu=2$ in (3.1). We use this choice in our numerical implementation in the example in $\S 4$.

Also, there is an additional motivation for choosing $\nu=2$ : With the choice $\nu=0$ (or $\nu=1$ ) we typically obtain (for each mode) two (or, resp., one) coefficient pairs $\left(b_{l}, q_{l}\right)$ of big magnitude. These "outlier" values reflect the different nature of the first two coefficients. Including them into our discrete sum of exponentials would then yield less accurate approximation results.

Let us fix $L$ and consider the formal power series

$$
g(x):=s^{(\nu)}+s^{(\nu+1)} x+s^{(\nu+2)} x^{2}+\ldots, \quad|x| \leq 1 .
$$

If the $[L-1 \mid L]$ Padé approximation

$$
\tilde{g}(x):=\frac{P_{L-1}(x)}{Q_{L}(x)}
$$

of (3.2) exists, then its Taylor series

$$
\tilde{g}(x)=\tilde{s}^{(\nu)}+\tilde{s}^{(\nu+1)} x+\tilde{s}^{(\nu+2)} x^{2}+\ldots
$$


satisfies the conditions

$$
\tilde{s}^{(n)}=s^{(n)}, \quad n=\nu, \nu+1, \ldots, 2 L+\nu-1,
$$

due to the definition of the Padé approximation rule.

TheOREm $3.2([10])$. Let $Q_{L}(x)$ have $L$ simple roots $q_{l}$ with $\left|q_{l}\right|>1, \quad l=1, \ldots, L$. Then

$$
\tilde{s}^{(n)}=\sum_{l=1}^{L} b_{l} q_{l}^{-n}, \quad n=\nu, \nu+1, \ldots
$$

where

$$
b_{l}:=-\frac{P_{L-1}\left(q_{l}\right)}{Q_{L}^{\prime}\left(q_{l}\right)} q_{l} \neq 0, \quad l=1, \ldots, L .
$$

REMARK 3.3. We remark that the assumption in Theorem 3.2 on the roots of $Q_{L}(x)$ to be simple is not essential. For multiple roots one only has to reformulate Theorem 3.2. All our practical calculations confirm that this assumption holds for any desired $L$, although we cannot prove this.

Evidently, the approximation to the convolution coefficients $s^{(n)}$ by the representation (3.1) using a $[L-1 \mid L]$ Padé approximant to (3.2) behaves as follows: the first $2 L$ coefficients are reproduced exactly; see (3.3). However, the asymptotics of $s^{(n)}$ and $\tilde{s}^{(n)}$ (as $\left.n \rightarrow \infty\right)$ differ strongly - algebraic versus exponential decay. The difference $\left\|s_{m}^{(n)}-\tilde{s}_{m}^{(n)}\right\|_{\ell_{n, m}^{2}}$ decreases strongly with respect to $L$; cf. Table 4.1 in $\S 4.2$. Note, however, that only the value of $L$ effectively chosen in the algorithm counts here (cf. $\S 4.2$ for details).

Fast evaluation of the discrete convolution. Let us consider the approximation (3.1) of the discrete convolution kernel appearing in the discrete TBC (2.40). With these "exponential" coefficients the approximated convolution

$$
\tilde{C}_{J}^{(n-1)}:=\sum_{p=1}^{n-1} \tilde{s}^{(n-p)} \psi_{J+1}^{(p)}, \quad \tilde{s}^{(n)}=\sum_{l=1}^{L} b_{l} q_{l}^{-n}, \quad\left|q_{l}\right|>1,
$$

of the discrete function $\psi_{J+1}^{(p)}, p=1,2, \ldots$ with the coefficients $\tilde{s}^{(n)}$ can be calculated by recurrence formulas, and this will reduce the numerical effort significantly.

A straightforward calculation (cf. [10]) yields:

$$
\tilde{C}_{J}^{(n-1)}=\sum_{l=1}^{L} \tilde{C}_{J, l}^{(n-1)}, \quad n \geq 2,
$$

where

$$
\begin{gathered}
\tilde{C}_{l}^{(0)} \equiv 0, \\
\tilde{C}_{J, l}^{(n-1)}=q_{l}^{-1} \tilde{C}_{J, l}^{(n-2)}+b_{l} q_{l}^{-1} \psi_{J+1}^{(n-2)},
\end{gathered}
$$

$n=2,3, \ldots, l=1, \ldots, L$. Finally we summarize the approach by the following algorithm. For each azimuth mode $m=0, \ldots, K-1$ : 
1. Calculate $\ell_{J+1}^{(n)}, n=0, \ldots, N-1$, with formulas (2.38) with a boundary value $\ell_{J_{\infty}}^{(n)}:=\ell_{\infty}^{(n)}$ that can be taken from the 1D plane case $\ell_{\infty}^{(n)} \equiv \ell^{(n)}$ from [10], and use (2.24) to find $s^{(n)}$.

2. Calculate $\tilde{s}^{(n)}$ via the Padé-algorithm.

3. The corresponding coefficients $b_{l}, q_{l}$ are used for the efficient calculation of the discrete convolutions.

REMARK 3.4. The sum-of-exponentials approximation of the discrete TBCs reduces the numerical effort drastically. The effort for the evaluation of the convolution sum $\sum_{p=1}^{n-1} s_{m}^{(n-p)} \psi_{J}^{(m, p)}$ is of order $\mathcal{O}\left(K n^{2}\right)$. With the proposed approximation of the discrete TBCs this can be reduced to linear effort $\mathcal{O}(K L n)$, where $L$ denotes the number of terms in the sum-of-exponentials (3.4). For using the exact discrete TBCs the convolution coefficients $s_{m}^{(n)}$ (cf. (2.24)) have to be calculated once in a set-up before the time-stepping for all modes $m$ and all time levels $p=0, \ldots, n$. As a further advantage of the approximated discrete TBCs, the coefficients (2.24) only need to be calculated for $p=0, \ldots, 2 L+\nu-1$ (cf. (3.3)).

\section{Numerical results}

In this section we present some numerical examples concerning the exact discrete TBCs and the approximated discrete TBCs. For further examples we refer the reader to $[48]$.

4.1. Exact discrete TBCs. Here we shall illustrate that our algorithm can compute the convolution coefficients of the TBC with almost machine accuracy. Hence, the (numerically computed) TBC in the circular case is essentially as accurate as in the rectangular case [10], where the TBCs are obtained analytically.

We recall the Example 2 from [26], i.e. we consider (1.1) with the vanishing potential $V \equiv 0$ and the angle-dependent initial data

$$
\psi^{I}(r, \theta)=\frac{e^{2 i k_{x} r \cos \theta+2 i k_{y} r \sin \theta-\frac{(r \cos \theta)^{2}}{2 \alpha_{x}}-\frac{(r \sin \theta)^{2}}{2 \alpha_{y}}}}{\sqrt{\alpha_{x} \alpha_{y}}} .
$$

Then the exact solution to (1.1a) for $t>0$ is given by the Gaussian beam

$$
\psi(r, \theta, t)=\frac{e^{2 i k_{x}\left(r \cos \theta-k_{x} t\right)+2 i k_{y}\left(r \sin \theta-k_{y} t\right)-\frac{\left(r \cos \theta-2 k_{x} t\right)^{2}}{2\left(\alpha_{x}+i t\right)}-\frac{\left(r \sin \theta-2 k_{y} t\right)^{2}}{2\left(\alpha_{y}+i t\right)}}}{\sqrt{\alpha_{x}+i t} \sqrt{\alpha_{y}+i t}} .
$$

We set the initial standard deviations $\alpha_{x}=\alpha_{y}=0.04$, let $k_{x}=1, k_{y}=-1$ and calculate a solution $\psi_{1}$ to (1.9) with an equidistant discretization on the circular domain $\Omega_{1}=$ $[0, R] \times[0,2 \pi]$ with $R=1$ and $J+2$ grid points in the $r$-direction and $K$ in the $\theta$ direction for the time interval $0<t \leq T=0.5$. In order to satisfy the assumption that the initial data is compactly supported in $\Omega_{1}$ (cf. $\S 2$ ) we use a small numerical cut-off close to $R, \psi^{I}(r, \theta)=0$ for $r \geq R-\Delta r$ for all angles $\theta$, i.e. in discrete notation $\psi_{j, k}^{0}=0$ for $j \geq J, k=0, \ldots, K-1$. We remark that this assumption of compactly supported initial data is not essential; strategies to overcome this restriction can be found in [22]. Since we use an offset grid, discrete TBCs are implemented as described before (cf. (2.40)-(2.41)) at $r=R-\Delta r / 2$, using the grid points $R$ and $R-\Delta r$. A reference solution $\psi_{2}$ is calculated on the domain $\Omega_{2}=[0,2 R] \times[0,2 \pi]$ with discrete TBCs at $r=2 R-\Delta r / 2$. To determine the error due to the PDE-scheme (1.9) we compare the 
numerical solution $\psi_{2}$ with the exact one $\psi$ on $\Omega_{1}$ : The relative $L^{2}$-error

$$
L_{\Omega_{1}}\left(\psi_{2}, \psi, t_{n}\right)=\frac{\left(\sum_{\left(r_{j}, \theta_{k}\right) \in \Omega_{1}} r_{j}\left|\psi_{2}\left(r_{j}, \theta_{k}, t_{n}\right)-\psi\left(r_{j}, \theta_{k}, t_{n}\right)\right|^{2}\right)^{\frac{1}{2}}}{\max _{0 \leq t_{n} \leq T}\left\{\left(\sum_{\left(r_{j}, \theta_{k}\right) \in \Omega_{1}} r_{j}\left|\psi\left(r_{j}, \theta_{k}, t_{n}\right)\right|^{2}\right)^{\frac{1}{2}}\right\}},
$$

is based on the norm defined in (2.5). This test includes also the error due to the cut-off of the initial function. The effects of the boundary should be negligible here, because the "main wave" of $\psi_{2}$ does not cross the boundary at $r=2 R$ during the considered time interval.

In order to distinguish between the error due to the difference scheme and the error due to the discrete TBCs, we compare the numerical solution $\psi_{1}$ with the numerical reference solution $\psi_{2}$ and calculate the relative error $L_{\Omega_{1}}\left(\psi_{2}, \psi_{1}, t_{n}\right)$ due to the boundary condition.

Discretization and results. The solutions $\psi_{1}$ and $\psi_{2}$ are calculated for three parameter sets. First we let $J=K=64$, i.e. $\Delta r=1 / 64, \Delta \theta=2 \pi / 64$, and $\Delta t=$ $1 / 64$, then $\Delta r=\Delta t=1 / 128, \Delta \theta=2 \pi / 128$, and finally $\Delta r=\Delta t=1 / 256, \Delta \theta=2 \pi / 256$. These discretization parameters are taken from [26]. The relative error of the initial function due to the cut-off is about $\mathcal{O}\left(10^{-6}\right), \mathcal{O}\left(10^{-7}\right), \mathcal{O}\left(10^{-7}\right)$, respectively. We present in Figure 4.1 the absolute value of the initial function (4.1) and of the evolution of the numerical solution $\psi_{1}$ on the computational domain $\Omega_{1}$ until $t=0.5$ for the last set of discretization parameters and the potential $V=0$. The Gaussian beam has an initial momentum specified by $k_{x}=1, k_{y}=-1$. As expected, the beam leaves the computational domain without any non-physical reflections at the artificial boundary. The observable broadening of the beam (as $t$ grows) is due to dispersive effects, which are equally present in the exact solution (4.2). Figure 4.2(a) shows the relative error $L_{\Omega_{1}}\left(\psi_{2}, \psi, t_{n}\right)$ of the numerical solution $\psi_{2}$ with respect to the exact solution restricted on $\Omega_{1}$ for the three sets of parameters: The scheme is second order in $\Delta r, \Delta \theta, \Delta t$.

The relative error $L_{\Omega_{1}}\left(\psi_{1}, \psi_{2}, t_{n}\right)$ due to the boundary condition is presented in Figure 4.2(b) also for all parameter sets. With values around $\mathcal{O}\left(10^{-13}\right)$ it is close to the rounding error of Matlab. So the error of our discrete TBC is negligible compared to the error of the finite difference scheme in the interior. In [26], however, the truncated TBCs introduce an additional error, which seems to be larger than the discretization error (cf. Fig. 10 in [26]).

We remark that the (small) error in Figure 4.2(b) consists of two contributions: The error of the discrete convolutions in the TBC which may slightly increase with a finer $t$-discretization - amounting to longer discrete convolutions. The second contribution stems from the iterative solver for the system of linear equations (cf. also Figure 4.3). Note that the spatial degrees of freedom increases by a factor four between each parameter set. Accordingly, the condition number of the system matrices for the three sets of parameters are about $5 \cdot 10^{4}, 4 \cdot 10^{5}, 3 \cdot 10^{6}$, respectively.

Our next test concerns the long-time behavior of the relative error due to the discrete TBCs. Therefore we calculate the numerical solutions $\psi_{1}, \psi_{2}$ of (1.9) for the initial data (4.1) with $k_{x}=k_{y}=0, \alpha_{x}=\alpha_{y}=0.5$ on the circular domains $\Omega_{1}=[0, R] \times$ $[0,2 \pi]$ and, resp., $\Omega_{2}=[0,2 R] \times[0,2 \pi]$ with $R=2.5$ until $t=4$. Since $k_{x}=k_{y}=0$, the beam does not propagate and only spreads due to dispersion. We use the three sets of discretization parameters $\Delta r=R / 64, \Delta \theta=2 \pi / 64 ; \Delta r=R / 128, \Delta \theta=2 \pi / 128$; and 


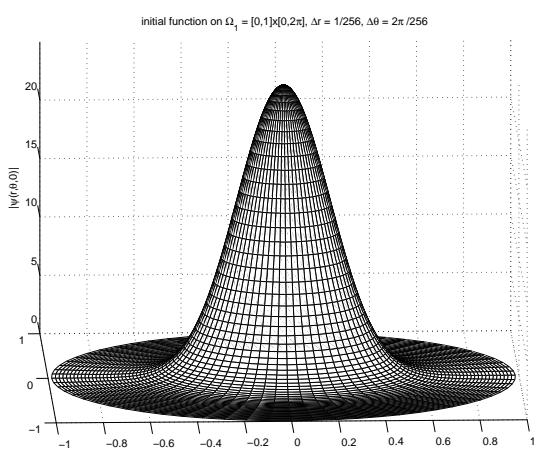

(a) $t=0$

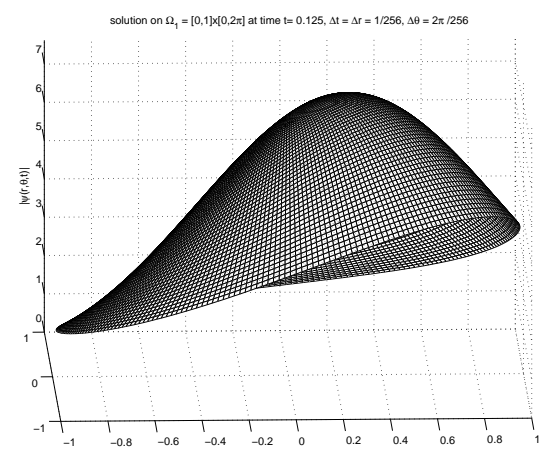

(c) $t=0.125$

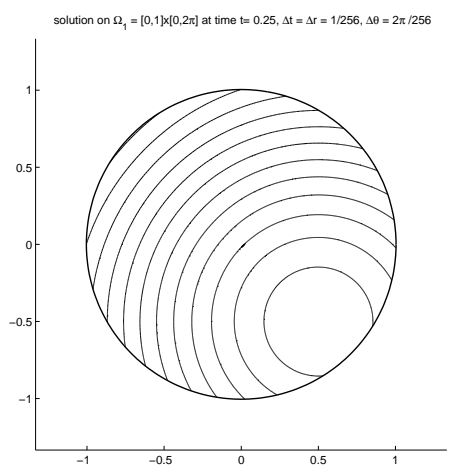

(e) $t=0.25$

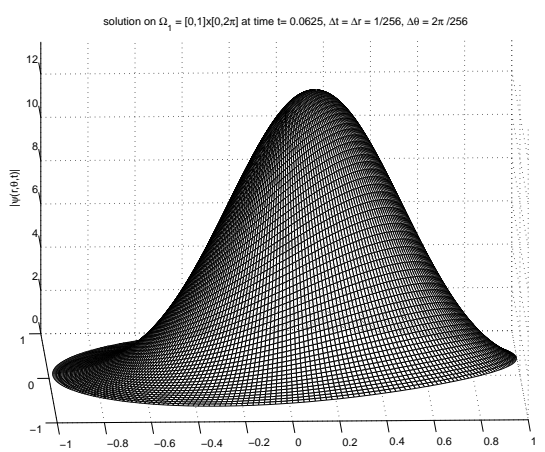

(b) $t=0.0625$

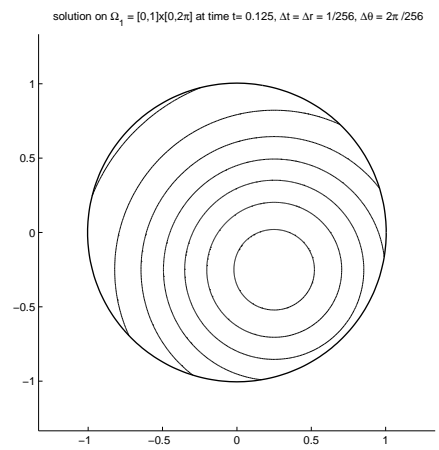

(d) $t=0.125$

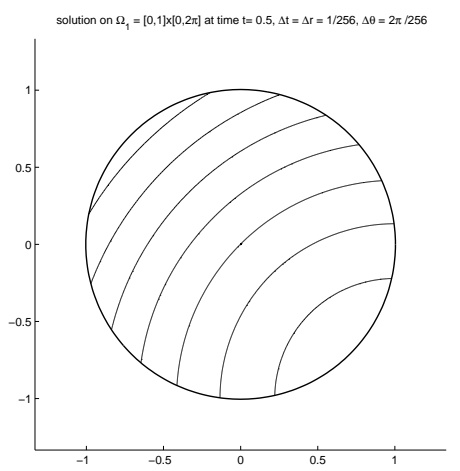

(f) $t=0.5$

FIG. 4.1. Absolute value (as normal/contour plots) of the initial function ((4.1) with cut-off) and the calculated solution $\psi_{1}$ of the scheme (1.9) on the computational domain $\Omega_{1}$ with $\Delta r=\Delta t=$ $1 / 256, \Delta \theta=2 \pi / 256, \alpha_{x}=\alpha_{y}=0.04$, and the wave numbers $k_{x}=1, k_{y}=-1 . V=0$; a discrete TBC is implemented at $r=1-\Delta r / 2$ : No reflections are visible.

$\Delta r=R / 256, \Delta \theta=2 \pi / 256$. For all calculations we let $\Delta t=0.01$. In Figure 4.3 we show the relative error $L_{\Omega_{1}}\left(\psi_{1}, \psi_{2}, t_{n}\right)$ due to the boundary conditions (and the residual of the solver for the linear system) for this long-time test. Observe that in this long-time calculation the error due to the discrete TBCs grows only sublinearly and is still only around $\mathcal{O}\left(10^{-13}\right)$. 


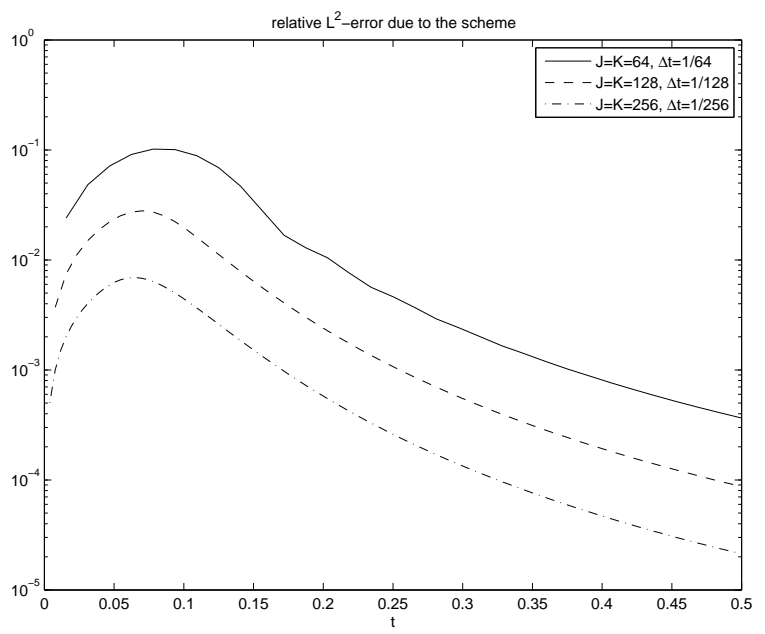

(a) error due to the scheme

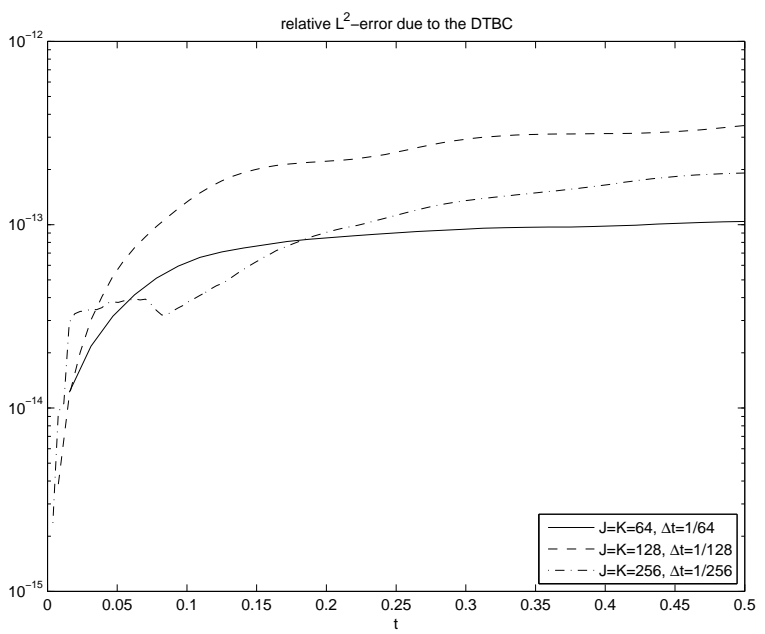

(b) error due to the boundary condition

FIG. 4.2. (a): Relative error $L_{\Omega_{1}}\left(\psi_{2}, \psi, t_{n}\right)$ due to the scheme and (b): relative error $L_{\Omega_{1}}\left(\psi_{1}, \psi_{2}, t_{n}\right)$ due to the boundary conditions. Both errors are calculated for the time evolution of initial function (4.1) for the three parameter sets with 64 (solid line), 128 (dashed line), and 256 grid points (dashed-dotted line).

In an additional numerical test (presented in $\S 4.4 .2$ of [48]) we also applied our discrete TBC-approach to the example of [5] (again $\psi^{I}$ from (4.1), but with modified parameters $k_{x}, k_{y}, \alpha_{x}, \alpha_{y}$ ). Since the authors of [5] only use (approximate) absorbing boundary conditions, their relative error is larger than with our discrete TBCapproach.

4.2. Approximated discrete TBCs. To illustrate the sum-of-exponential ansatz we consider again the initial function (4.1) with the parameters $\alpha_{x}=\alpha_{y}=0.04$ and $k_{x}=-k_{y}=1$. We consider the initial data (4.1) with a cut-off at $R-\Delta r$, which 


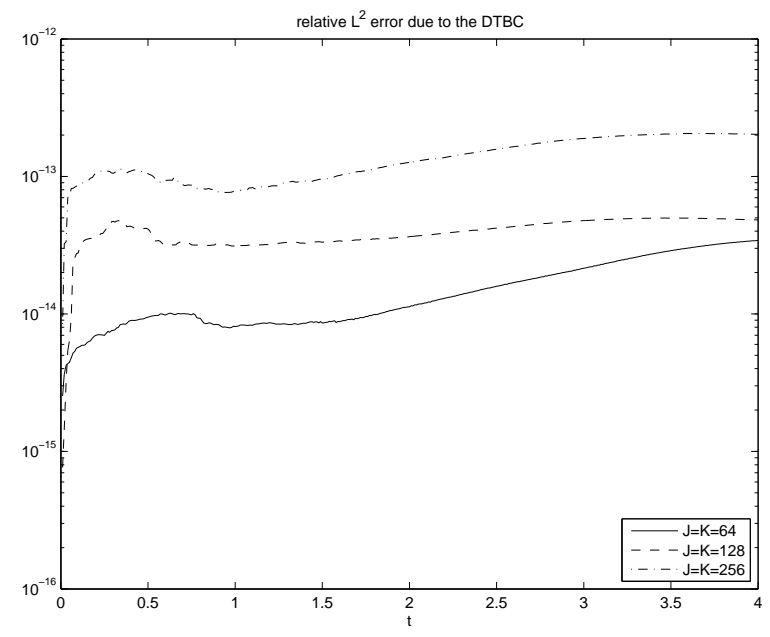

FIG. 4.3. Relative error $L_{\Omega_{1}}\left(\psi_{1}, \psi_{2}, t_{n}\right)$ due to the boundary condition for the time evolution of initial function (4.1) with $k_{x}=k_{y}=0, \alpha_{x}=\alpha_{y}=0.5$ for the three parameter sets with 64 (solid line), 128 (dashed line), and 256 grid points (dashed-dotted line). The time step size equals $\Delta t=0.01$.

causes a relative error of the order $\mathcal{O}\left(10^{-6}\right)$. With this initial data a solution $\psi_{1}$ of (1.9) is calculated on the circular domain $\Omega_{1}=[0, R] \times[0,2 \pi]$ with the radius $R=1$. For the discrete TBCs we use the approximation (3.6). As a numerical reference solution we take $\psi_{2}$, which is obtained with the exact discrete TBCs (2.41) on the larger domain $[0,2] \times[0,2 \pi]$.

Discretization and results. For the discretization parameters $\Delta t=0.002, \Delta r=$ $1 / 64, \Delta \theta=2 \pi / 64$ we evolve the solution up to $t=0.5$. In the sum-of-exponentials we choose $\nu=2$ in the three different calculations, $L=10,20$, and 40 . We obtain the first $2 L+\nu-1$ convolution coefficients exactly by the recursion formula (2.38) with an initial value $\ell_{J_{\infty}, m}^{(n)}:=\ell_{\infty, m}^{(n)}$ taken from the $1 \mathrm{D}$ plane case $\ell_{\infty, m}^{(n)} \equiv \ell_{m}^{(n)}$ from [10] for each mode $m=0, \ldots, K-1$ and sum them according to (2.24). The sets $\left\{b_{l, m}, q_{l, m}\right\}$, $l=1, \ldots, L$ needed for the calculation of the approximated convolution coefficients $\tilde{s}_{m}^{(n)}$, $n>2 L+\nu-1$ for all modes $m$ are obtained by the Padé algorithm described in $\S 3$. We realized these calculations by a Maple code, within which we try to find $L$ roots $q_{l, m}$ of the polynomial $Q_{L}(x)$ as it is described in Theorem 3.2 (separately for each mode). Due to a "nearly breakdown" by ill conditioned steps in the Lanczos algorithm (cf. [16]) it is not always possible to find $L$ roots of $Q_{L, m}$ fulfilling the condition $\left|q_{l, m}\right|>1, l=1, \ldots, L$ for each mode $m=0, \ldots, K-1$. Consequently, the Maple code automatically chooses smaller and smaller values $(L-1, L-2, \ldots)$ to guarantee that all roots have an absolute value larger than 1 . E.g., with the initial choice $L=40$ you will find values for $L$ fulfilling the above condition that vary from 18 to 32 for the different modes. The number of summands $L$ is hence just an initial guess for the final number of summands in the sum-of-exponentials; cf. Table 4.1.

In Figure 4.4 we present the contour plots of the absolute value of the solution $\psi_{1}$ at time $t=0.5$, calculated with the approximated discrete TBCs with $L=10$ and $L=40$ terms in the sum-of-exponentials. For $L=10$ there are strong non-physical reflections (see Figure 4.4(a)), for larger values of $L$ these reflections become invisible 


\begin{tabular}{|c|c|c|c|}
\hline initial $L$ & 10 & 20 & 30 \\
\hline$\left\|s_{m}^{(n)}-\tilde{s}_{m}^{(n)}\right\|_{\ell_{n, m}^{2}}$ & $2.75 \mathrm{e}-04$ & $1.61 \mathrm{e}-05$ & $1.32 \mathrm{e}-05$ \\
\hline$L_{\min }$ & 5 & 14 & 14 \\
\hline$L_{\max }$ & 10 & 20 & 30 \\
\hline
\end{tabular}

TABLE 4.1. $L^{2}$-errors in the approximated coefficients $\tilde{s}_{m}^{(n)}$ for different initial choices of L. $L_{\min }, L_{\max }$ are the effectively used smallest and largest numbers of summands with respect to the 64 modes.

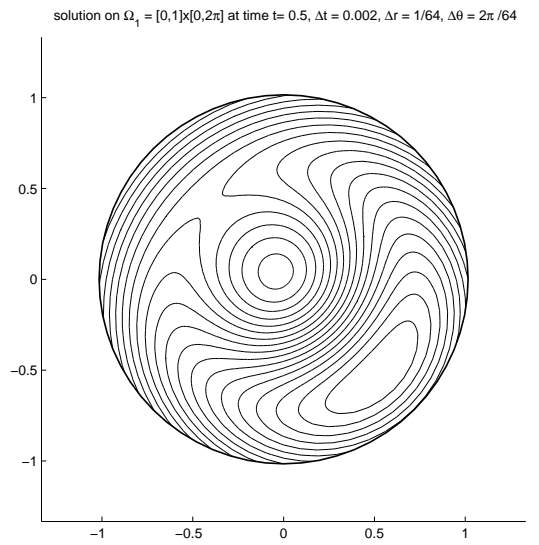

(a) $L=10$

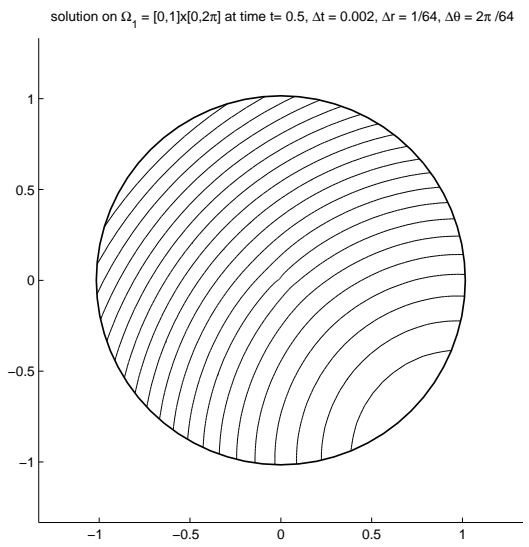

(b) $L=40$

FIG. 4.4. Contour plots of the absolute value of the calculated solution $\psi_{1}$ of (1.9) at $t=0.5$ with the initial function (4.1) on the computational domain $\Omega_{1}$. We use approximated discrete TBCs with the initial choices (a) $L=10$ and (b) $L=40$.

(see Figure 4.4(b)).

The relative $L^{2}$-error due to the approximated discrete TBCs is shown in Figure 4.5. For different initial choices of the number of coefficients $L$ in the sum-ofexponentials we present the error $L_{\Omega_{1}}\left(\psi_{1}, \psi_{2}, t_{n}\right)$ (cf. (4.3)) there. Although the coefficients $s_{m}^{(n)}, \tilde{s}_{m}^{(n)}$ have different asymptotic behaviors (algebraic vs. exponential decay) the error grows only moderately in time. In our next test we show that long time calculations with the approximated discrete TBCs are stable. To this end we evolve the initial data (4.1) with $\alpha_{x}=\alpha_{y}=0.04$ and $k_{x}=k_{y}=0$ up to $t=20$. We use the discretization parameters $\Delta t=0.002, \Delta r=1 / 64, \Delta \theta=2 \pi / 64$ for different initial choices for $L$, the number of summands. Due to dispersion the norm of the solution decays in time, as it is shown in Figure 4.6.

In $\S 4.4 .3$ of [48] we applied the sum-of-exponentials approximation to an additional numerical example and compared it to the example from [31] (again $\psi^{I}$ from (4.1), but with modified parameters $k_{x}, k_{y}, \alpha_{x}, \alpha_{y}$ ). In [31] the authors presented a different sum-of-exponentials approximation for the convolution kernel of the TBC for the 2D Schrödinger equation.

We remark that the sum-of-exponentials approach for discrete TBCs of the 1D Schrödinger equation (including the Maple code) is presented at http://www-amna.math.uni-wuppertal.de/ ${ }^{\sim}$ ehrhardt/dtbc.html. 


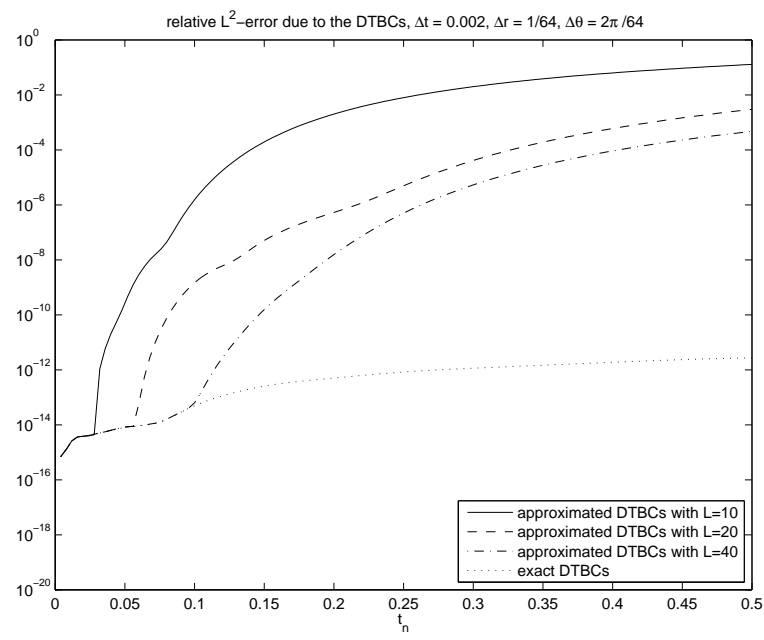

FIG. 4.5. Relative error $L_{\Omega_{1}}\left(\psi_{1}, \psi_{2}, t_{n}\right)$ due to the approximated discrete TBCs as a function of $t_{n} \in[0,0.5]$ for the time evolution of the initial function (4.1) for different initial choices of the number $L$ in the sum-of-exponentials, 10 (solid line), 20 (dashed line), and 40 grid points (dasheddotted line). The relative error due to the exact discrete TBCs for this problem is plotted in the dotted line.

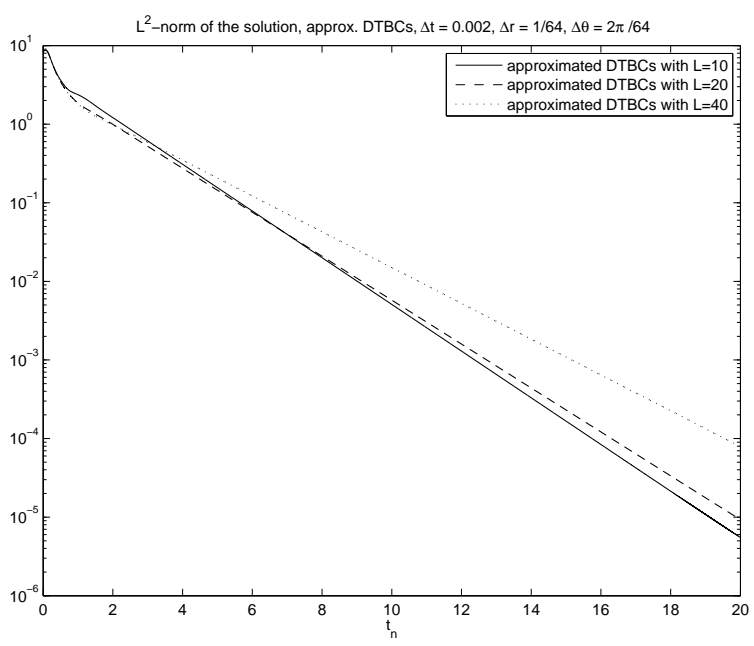

FIG. 4.6. $L^{2}$-norm for the long time evolution of the initial function (4.1) with $\alpha_{x}=\alpha_{y}=$ 0.04 and $k_{x}=k_{y}=0$ again for different initial choices of the number $L$ in the sum-of-exponentials, 10 (solid line), 20 (dashed line), and 40 grid points (dotted line). We choose the discretization parameters $\Delta r=1 / 64, \Delta \theta=2 \pi / 64, \Delta t=0.002$.

\section{Conclusion and future work}

In this paper we extend the ideas and the fast evaluation technique of the paper $[10]$ to the case of a two-dimensional circular domain. We proved a discrete energy conservation for the proposed Crank-Nicolson type finite difference scheme. After a Z-transformation with respect to the time level we considered for each angular mode 
a variable-coefficient Riccati difference equation to derive the discrete TBC. Then we proposed and proved a stable algorithm to compute the convolution coefficients for each angular mode. We conclude with several numerical examples illustrating that the new TBC yields accurate results.

Concerning possible extensions of the present work we note that the Schrödinger equation on elliptical domains is being used for simulations of diatomic molecules [55] and ellipsoidal quantum dots [17, 34], for example. Hence, it is an interesting future research direction to further generalize our approach to ellipsoidal domains. In this respect we note that, for $V_{R}<0$, the Laplace transformed Schrödinger Equation (1.3) can be regarded as a Helmholtz-type equation in polar coordinates with a complex wave number. Such equations can be solved by Mathieu functions for elliptical shaped boundaries; cf. [12, 14, 39, 40, 42] and the references therein.

A second challenging future work direction would be to generalize our method to 3 space dimensions. For the continuous TBC this was done in [27] and recently by Heinen and Kull in $[28,29]$ by using a spherical harmonic expansion. Hence, it seems feasible that our strategy can also be extended to the discrete TBC in 3D. However, the inclusion of the latitudinal differential operator brings about new features (like the singularity of the coefficients and discrete BCs at the poles) that will have to be checked in detail.

Appendix A. New discrete TBCs for the 1D Schrödinger equation. With a uniform spatial discretization, the Crank-Nicolson finite difference scheme for the Schrödinger equation with constant potential $V$ reads:

$$
\begin{array}{r}
-\frac{2 i}{\Delta t}\left(\psi_{j}^{(n+1)}-\psi_{j}^{(n)}\right)=\frac{\psi_{j+1}^{\left(n+\frac{1}{2}\right)}-2 \psi_{j}^{\left(n+\frac{1}{2}\right)}+\psi_{j-1}^{\left(n+\frac{1}{2}\right)}}{\Delta x^{2}}-2 V \psi_{j}^{\left(n+\frac{1}{2}\right)}, \\
j \in \mathbb{Z}, n \in \mathbb{N}_{0} .
\end{array}
$$

Let the index interval $j=0, \ldots, J$ denote the computational domain, and abbreviate the mesh ratio by $\rho:=4 \Delta x^{2} / \Delta t$. Using the $Z$-transform method of $\S 2$ (or [7]) one first derives the discrete TBC for the $Z$-transformed variable:

$$
\hat{\psi}_{J}(z)=\hat{\ell}(z) \hat{\psi}_{J-1}(z),
$$

with $\hat{\ell}(z)$ given by $(2.22)$, if replacing there $V_{R}$ by $V$. As before, we choose the branch of the square root such that $|\ell(z)| \leq 1$ for $z \geq 1$. An inverse $Z$-transformation of (A.1) yields (similarly to $[22, \S 3.2]$ ):

$$
\psi_{J}^{(n)}=\ell^{(n)} * \psi_{J-1}^{(n)} .
$$

or explicitly (when assuming $\psi_{j}^{(0)}=0, j \geq J-1$ )

$$
\psi_{J}^{(n)}-\ell^{(0)} \psi_{J-1}^{(n)}=\sum_{p=1}^{n-1} \ell^{(n-p)} * \psi_{J-1}^{(p)} .
$$

Here, the convolution coefficients are given by

$$
\begin{aligned}
\ell^{(n)}=\left[1+i \frac{\rho}{2}+\frac{\sigma}{2}\right] \delta_{n}^{0}-i \rho(-1)^{n}+\frac{i}{2} \sqrt[4]{\left(\rho^{2}+\sigma^{2}\right)\left[\rho^{2}+(\sigma+4)^{2}\right]} e^{-i \varphi / 2} \\
\cdot e^{-i n \varphi}\left\{\lambda P_{n}(\mu)+P_{n-1}(\mu)+\tau \sum_{k=0}^{n-1}(-\lambda)^{n-k} P_{k}(\mu)\right\}
\end{aligned}
$$


with $\kappa=V \Delta t / 2, \sigma=\rho \kappa, \delta$ the Kronecker delta, $P_{n}$ the Legendre polynomials, and $P_{-1} \equiv 0$. Here we used the following abbreviations:

$$
\lambda:=\frac{\rho-4 \kappa-\rho \kappa^{2}+2 i(\rho \kappa+2)}{\sqrt{\left(1+\kappa^{2}\right)\left[\rho^{2}+(\rho \kappa+4)^{2}\right]}}=e^{i \varphi}, \text { with } \varphi=\arctan \frac{2(\rho \kappa+2)}{\rho-4 \kappa-\rho \kappa^{2}} .
$$

Moreover

$$
\begin{gathered}
\mu:=\frac{\rho\left(1+\kappa^{2}\right)+4 \kappa}{\sqrt{\left(1+\kappa^{2}\right)\left[\rho^{2}+(\rho \kappa+4)^{2}\right]}} \in(-1,1), \\
\tau:=\frac{4 \rho}{\sqrt{\left(1+\kappa^{2}\right)\left[\rho^{2}+(\rho \kappa+4)^{2}\right]}} \in \mathbb{R} .
\end{gathered}
$$

Note that the convolution in (A.2) is implemented at the grid point $J$-1, i.e. at the interior of the two boundary grid points $J-1, J$. This is in contrast to the discrete $\mathrm{TBC}$ in [7], which uses a convolution on the exterior boundary grid point:

$$
\psi_{J-1}^{(n)}=\tilde{\ell}^{(n)} * \psi_{J}^{(n)} .
$$

There, the convolution coefficients $\tilde{\ell}^{(n)}$ are defined with the opposite sign in front of the fourth root of (A.4).

This slight reformulation of the discrete TBCs has an important practical consequence: While the coefficients $\tilde{\ell}^{(n)}$ are oscillatory $\left(\tilde{\ell}^{(n)} \approx 2 i \rho(-1)^{n}\right.$, cf. [22, S3.3]), the coefficients $\ell^{(n)}$ decay like $n^{-3 / 2}$. Hence, this new formulation (A.2) does not require us to introduce the "summed convolution coefficients" of [7, 22].

A more refined asymptotic of the coefficients $\ell^{(n)}$ is given in (2.23).

Acknowledgment. A. A. acknowledges partial support from the FWF (Wissenschaftskolleg Differentialgleichungen and project I 395-N16). The first two authors were partially supported by the DFG under Grant-No. AR 277/3-3. The last three authors were supported by the DFG Research Center MATHeon "Mathematics for key technologies" in Berlin. The fourth author was also supported by RFBR-Grant No. 10-01-00567.

\section{REFERENCES}

[1] I. Alonso-Mallo and N. Reguera, Weak ill-posedness of spatial discretizations of absorbing boundary conditions for Schrödinger-type equations, SIAM J. Numer. Anal., 40, 134-158, 2002.

[2] B. Alpert, L. Greengard, and T. Hagstrom, Rapid evaluation of nonreflecting boundary kernels for time-domain wave propagation, SIAM J. Numer. Anal., 37, 1138-1164, 2000.

[3] B. Alpert, L. Greengard, and T. Hagstrom, Nonreflecting boundary conditions for the timedependent wave equation, J. Comput. Phys., 180, 270-296, 2002.

[4] X. Antoine and C. Besse, Unconditionally stable discretization schemes of non-reflecting boundary conditions for the one-dimensional Schrödinger equation, J. Comput. Phys., 188, 157$175,2003$.

[5] X. Antoine, C. Besse, and V. Mouysset, Numerical Schemes for the simulation of the twodimensional Schrödinger equation using non-reflecting boundary conditions, Math. Comput., 73, 1779-1799, 2004.

[6] X. Antoine, A. Arnold, C. Besse, M. Ehrhardt, and A. Schädle, A review of transparent and artificial boundary conditions techniques for linear and nonlinear Schrödinger equations, Commun. Comput. Phys., 4, 729-796, 2008. 
[7] A. Arnold, Numerically absorbing boundary conditions for quantum evolution equations, VLSI Design, 6, 313-319, 1998.

[8] A. Arnold and M. Ehrhardt, Discrete transparent boundary conditions for wide angle parabolic equations in underwater acoustics, J. Comput. Phys., 145, 611-638, 1998.

[9] A. Arnold, Mathematical concepts of open quantum boundary conditions, Transp. Theory Stat. Phys., 30, 561-584, 2001.

[10] A. Arnold, M. Ehrhardt, and I. Sofronov, Discrete transparent boundary conditions for the Schrödinger equation: Fast calculation, approximation, and stability, Commun. Math. Sci., 1, 501-556, 2003.

[11] A. Arnold, M. Ehrhardt, and M. Schulte, Numerical simulation of quantum waveguides, in VLSI and Computer Architecture, Nova Science Publishers, K. Watanabe (ed.), Inc., Hauppauge, NY 11788, 115-137, 2009.

[12] H. Barucq, R. Djellouli, and A. Saint-Guirons, Performance assessment of a new class of local absorbing boundary conditions for elliptical- and prolate spheroidal-shaped boundaries, Appl. Numer. Math., 59, 1467-1498, 2009.

[13] V.A. Baskakov and A.V. Popov, Implementation of transparent boundaries for numerical solution of the Schrödinger equation, Wave Motion, 14, 123-128, 1991.

[14] A. Bayliss, M. Gunzburger, and E. Turkel, Boundary conditions for the numerical solution of elliptic equations in exterior regions, SIAM J. Appl. Math., 42, 430-451, 1982.

[15] C.H. Bruneau and L. Di Menza, Conditions aux limites transparentes et artificielles pour l'équation de Schrödinger en dimension 1 d'espace, C.R. Acad. Sci. Paris, Ser. I, 320, 89-94, 1995.

[16] A. Bultheel and M. Van Barel, Linear Algebra, Rational Approximation and Orthogonal Polynomials, Studies in Comput. Math., North-Holland, 6, 1997.

[17] G. Cantele, D. Ninno, and G. Iadonisi, Confined states in ellipsoidal quantum dots, J. Phys. Condens. Matter, 12, 9019-9036, 2000.

[18] J.F. Claerbout, Coarse grid calculation of waves in inhomogeneous media with application to delineation of complicated seismic structure, Geophysics, 35, 407-418, 1970.

[19] A. Dedner, D. Kröner, I. Sofronov, and M. Wesenberg, Transparent boundary conditions for MHD simulations in stratified atmospheres, J. Comput. Phys., 171, 448-478, 2001.

[20] G. Doetsch, Anleitung Zum Praktischen Gebrauch der Laplace-Transformation und der ZTransformation, R. Oldenburg Verlag München, Wien, Auflage, 3, 1967.

[21] M. Ehrhardt, Discrete Artificial Boundary Conditions, Ph.D. Thesis, Technische Universität Berlin, 2001.

[22] M. Ehrhardt and A. Arnold, Discrete transparent boundary conditions for the Schrödinger equation, Riv. Mat. Univ. Parma, 6, 57-108, 2001.

[23] M. Ehrhardt, Discrete transparent boundary conditions for Schrödinger-type equations for non-compactly supported initial data, Appl. Numer. Math., 58, 660-673, 2008.

[24] L. Greengard and J. Strain, A fast algorithm for the evaluation of heat potentials, Commun. Pure Appl. Math., 43, 949-963, 1990.

[25] T. Hagstrom, Radiation boundary conditions for the numerical simulation of waves, Acta Numerica, 8, 47-106, 1999.

[26] H. Han and Z. Huang, Exact artificial boundary conditions for the Schrödinger equation in $\mathbb{R}^{2}$, Commun. Math. Sci., 2, 79-94, 2004.

[27] H. Han, D. Yin, and Z. Huang, Numerical solutions of Schrödinger equations in $\mathbb{R}^{3}$, Numer. Meth. Par. Diff. Eqs., 23, 511-533, 2007.

[28] M. Heinen and H.J. Kull, Numerical calculation of strong-field laser-atom interaction: An approach with perfect reflection-free radiation boundary conditions, Laser Physics, 20, 581$590,2010$.

[29] M. Heinen and H.J. Kull, Radiation boundary conditions for the numerical solution of the threedimensional time-dependent Schrödinger equation with a localized interaction, Physical Review E, 79, 056709, 2010.

[30] W. Huang, C. Xu, S.T. Chu, and S.K. Chaudhuri, The finite-difference vector beam propagation method: Analysis and assessment, J. Lightwave Technology, 10, 295-304, 1992.

[31] S. Jiang and L. Greengard, Efficient representation of nonreflecting boundary conditions for the time-dependent Schrödinger equation in two dimensions, Commun. Pure Appl. Math., 61, 261-288, 2008.

[32] G.E. Keiser, Optical Fiber Communication, McGraw-Hill, New York, 1983.

[33] M.R.S. Kulenović and G. Ladas, Dynamics of Second Order Rational Difference Equations, Chapman \& Hall, 2002.

[34] L.C. Lew Yan Voon and M. Willatzen, On triaxial ellipsoidal quantum dots, J. Phys. Condens. Matter, 16, 1087-1093, 2004. 
[35] M.F. Levy, Non-local boundary conditions for radiowave propagation, in Third International Conference on Mathematical and Numerical Wave Propagation Phenomena, Juan-les-Pins, France, 24-28, 1995.

[36] M.F. Levy, Parabolic equation models for electromagnetic wave propagation, IEE Electromagnetic Waves Series 45, IET, 2000.

[37] C. Lubich and A. Schädle, Fast convolution for non-reflecting boundary conditions, SIAM J. Sci. Comput., 24, 161-182, 2002.

[38] B. Mayfield, Non-Local Boundary Conditions for the Schrödinger Equation, Ph.D. Thesis, University of Rhode Island, Providence, RI, 1989.

[39] M. Medvinsky, E. Turkel, and U. Hetmaniuk, Local absorbing boundary conditions for elliptical shaped boundaries, J. Comput. Phys., 227, 8254-8267, 2008.

[40] M. Medvinsky and E. Turkel, On surface radiation conditions for an ellipse J. Comput. Appl. Math., 234, 1647-1655, 2010.

[41] S. Özbayat, and R. Janaswamy, Effective local absorbing boundary conditions for a finite difference implementation of the parabolic equation, IEEE Transactions on Antennas and Propagation, 59, 1616-1625, 2011.

[42] R.C. Reiner, R. Djellouli, and I. Harari, The performance of local absorbing boundary conditions for acoustic scattering from elliptical shapes, Comput. Meth. Appl. Mech. Engrg., 195, 3622-3665, 2006.

[43] A. Schädle, Numerische Behandlung transparenter Randbedingungen für die SchrödingerGleichung, Diploma Thesis, Universität Tübingen, 1998.

[44] A. Schädle, Non-reflecting boundary conditions for the two dimensional Schrödinger equation, Wave Motion, 35, 181-188, 2002.

[45] F. Schmidt and P. Deuflhard, Discrete transparent boundary conditions for the numerical solution of Fresnel's equation, Comput. Math. Appl., 29, 53-76, 1995.

[46] F. Schmidt, Construction of discrete transparent boundary conditions for Schrödinger-type equations, Surv. Math. Ind., 9, 87-100, 1999.

[47] M. Schulte, Transparente Randbedingungen für die Schrödinger Gleichung, Diploma Thesis, Westfälische Wilhelms-Universität Münster, 2004.

[48] M. Schulte, Numerical Solution of the Schrödinger Equation on Unbounded Domains, Dissertation, Westfälische Wilhelms-Universität Münster, 2007.

[49] M. Schulte and A. Arnold, Discrete transparent boundary conditions for the Schrödinger equation - A compact higher order scheme, Kinetic and Related Models, 1, 101-125, 2008.

[50] I.L. Sofronov, Conditions for complete transparency on the sphere for the three-dimensional wave equation, Russian Acad. Sci. Dokl. Math., 46, 397-401, 1993.

[51] I.L. Sofronov, Artificial boundary conditions of absolute transparency for two- and threedimensional external time-dependent scattering problems, Euro. J. Appl. Math., 9, 561$588,1998$.

[52] I.L. Sofronov, Non-reflecting inflow and outflow in wind tunnel for transonic time-accurate simulation, J. Math. Anal. Appl., 221, 92-115, 1998.

[53] J. Szeftel, Design of absorbing boundary conditions for Schrödinger equations in $\mathbb{R}^{d}$, SIAM J. Numer. Anal., 42, 1527-1551, 2004.

[54] J. Szeftel, Absorbing boundary conditions for nonlinear scalar partial differential equations, Comput. Methods Appl. Mech. Engrg., 195, 3760-3775, 2006.

[55] L. Tao, C.W. McCurdy, and T.N. Rescigno, Grid-based methods for diatomic quantum scattering problems. III. Double photoionization of molecular hydrogen in prolate spheroidal coordinates, Phys. Rev. A, 82, 023423, 2010.

[56] F.D. Tappert, The parabolic approximation method, in Wave Propagation and Underwater Acoustics, Lecture Notes in Physics 70, eds. J.B. Keller and J.S. Papadakis, Springer, New York, 224-287, 1977.

[57] C. Vassalo, Optical Waveguide Concepts, Elsevier, 1991.

[58] B. Zamzow, Simulation von Glasfaserspleißen mit der Beam-Propagation-Methode, Fortschritt-Berichte VDI Reihe 10, Nr. 655, Düsseldorf, VDI Verlag, 2001.

[59] C. Zheng, A perfectly matched layer approach to the nonlinear Schrödinger wave equations, J. Comput. Phys., 227, 537-556, 2007.

[60] A. Zisowsky, Discrete Transparent Boundary Conditions for Systems of Evolution Equations, Ph.D. Thesis, Technische Universität Berlin, 2003.

[61] A. Zisowsky and M. Ehrhardt, Discrete artificial boundary conditions for nonlinear Schrödinger equations, Math. Comput. Modell., 47, 1264-1283, 2008. 